\title{
1 Dual roles for LUBAC signaling in thymic epithelial cell development and survival
}

3 Reema Jain ${ }^{1,2, ~ \#, ~ K e l i n ~ Z h a o ~}{ }^{1,2}$, Julie M. Sheridan ${ }^{1,2}$, Melanie Heinlein ${ }^{1,2^{*}}$, Fiona Kupresanin ${ }^{1 \wedge}$,

4 Waruni Abeysekera ${ }^{1,2}$, Cathrine Hall ${ }^{1,2}$, James Rickard ${ }^{1,2}$, Philippe Bouillet ${ }^{1,2}$, Henning

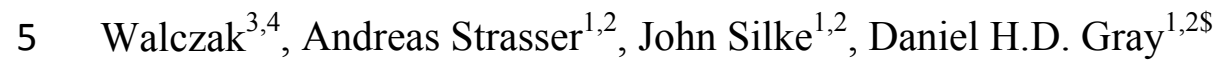

$6 \quad{ }^{1}$ Walter and Eliza Hall Institute of Medical Research, Melbourne, VIC, Australia; ${ }^{2}$ Department

7 of Medical Biology, University of Melbourne, Melbourne, VIC, Australia; ${ }^{3}$ Centre for Cell

8 Death, Cancer and Inflammation, UCL Cancer Institute, University College London, London,

$9 \mathrm{UK},{ }^{4}$ Centre for Biochemistry, University of Cologne, Cologne, Germany

$10{ }^{\#}$ Current address Fred Hutchinson Cancer Research Center, Seattle, Washington, USA.

$11 *$ Current address Department of Molecular Oncology, Genentech, Inc., South San Francisco,

12 CA, USA

$13 \wedge$ Current address ANZAC Research Institute, Concord, Australia

14

15 \$Correspondence to: $\underline{\text { dgray@,wehi.edu.au }}$

16

17 T: +61393452741

$18 \quad \mathrm{~F}:+61393470852$

19

20 Short Title: Essential functions for LUBAC in thymic epithelium

21

22 


\section{Abstract}

24 Thymic epithelial cells (TECs) form a unique microenvironment that orchestrates $\mathrm{T}$ cell

25 differentiation and immunological tolerance. Despite the importance of TECs for adaptive

26 immunity, there is an incomplete understanding of the signalling networks that support their

27 differentiation and survival. We report that the linear ubiquitin chain assembly complex

28 (LUBAC) is essential for medullary TEC (mTEC) differentiation, cortical TEC survival and

29 prevention of premature thymic atrophy. TEC-specific loss of LUBAC proteins, HOIL-1 or

30 HOIP, severely impaired expansion of the thymic medulla and AIRE-expressing cells.

31 Furthermore, HOIL-1-deficiency caused early thymic atrophy due to Caspase-8/MLKL-

32 dependent apoptosis/necroptosis of cortical TECs. By contrast, deficiency in the LUBAC

33 component, SHARPIN, caused relatively mild defects only in mTECs. These distinct roles for

34 LUBAC components in TECs correlate with their function in linear ubiquitination, NFKB

35 activation and cell survival. Thus, our findings reveal dual roles for LUBAC signaling in TEC

36 differentiation and survival. 


\section{Introduction}

39 The differentiation of haematopoietic progenitors into naive $\mathrm{T}$ cells in the thymus is governed by

40 thymic epithelial cells (TECs). Specialized TEC subtypes direct distinct quality control processes

41 in thymocyte differentiation. Cortical thymic epithelial cells (cTECs) mediate early events,

42 including $\mathrm{T}$ cell lineage commitment, proliferation and positive selection of cells expressing

43 TCRs capable of interacting with self-peptide/MHC complexes (1). By contrast, medullary

44 thymic epithelial cells (mTECs) are important for thymic negative selection and the generation

45 of FOXP3 $^{+}$regulatory $\mathrm{T}$ (Treg) cells, thus limiting the risk of autoimmunity (2). Medullary TECs

46 are uniquely adapted for the induction of immunological tolerance because they express

47 thousands of tissue-specific antigens that greatly increase the scope of thymic negative selection.

48 This property is mediated in mTEC subtypes by the transcriptional regulators AIRE and FEZF2

$49(2,3)$. These essential functions of TEC in immunity and tolerance have generated considerable

50 interest in the molecular mechanisms of their differentiation and maintenance.

51

52 Members of the tumour necrosis factor (TNF) and TNF receptor (TNFR) superfamilies (i.e. the

53 TNFSF and TNFRSF) and NF- $\kappa B$ transcription factors are critical for TEC differentiation and

54 the establishment of thymic tolerance (4). Signalling through RANK is required for mTECs

55 during development, whereas signals from other TNFRSF members co-ordinate the maintenance

56 of postnatal mTECs $(5,6)$. Ligation of the TNFRSF members CD40, RANK and lymphotoxin

57 beta receptor (LT $\beta R$ ) is required for the development of the key tolerogenic mTEC populations,

58 such as AIRE $^{\text {pos }}$ and FEZF2 ${ }^{\text {pos }}$ cells $(3,5,7-9)$. Yet, precisely how these signals are integrated to

59 direct TEC fate, function and survival remains poorly understood.

60 
61 LUBAC is a component of TNFR1 and CD40 receptor signalling complexes $(10,11)$ that

62 attaches linear ubiquitin chains to signal transducers and/or regulators of the canonical NF- $\kappa \mathrm{B}$

63 pathway, including RIPK1, TRADD, NEMO and TNFR1 itself $(10,12,13)$. LUBAC is a $\sim 600-$

$64 \mathrm{kD}$ ubiquitin E3 complex composed of three proteins: SHANK-associated RH domain

65 interacting protein (SIPL1/SHARPIN), C3HC4-type zinc finger containing 1 (RBCK1/HOIL-1)

66 and the catalytic component, ring finger protein 31 (RNF31/HOIP) $(10,12,14-16)$. Mutations of

67 these LUBAC components perturb innate and adaptive immune responses $(10,17,18)$. Patients

68 with loss-of-function mutations in HOIL-1 or HOIP were found to be T cell deficient and (in one

69 patient) had greatly reduced T-cell receptor excision circles, indicating impaired thymic function $70(17,18)$.

71

72 There is a differential requirement for HOIP, HOIL-1 and SHARPIN for LUBAC function,

73 signal transduction, differentiation and cell death. Deficiency in HOIP or HOIL-1 completely

74 abolishes LUBAC activity, impairs NF-אB activation and promotes cell death (10, 15, 19-21).

75 By contrast, SHARPIN-deficient cells can carry out diminished linear ubiquitination via HOIL-

76 1/HOIP complexes, attenuated activation of NF- $\mathrm{BB}$ and JNK signaling pathways and are also

77 sensitized to cell death (10, 21-23). Importantly, LUBAC functions in NF- $\mathrm{BB}$ activation and cell

78 survival can be independent (24).

79

80 The in vivo consequences of these defects vary according to cell type and developmental context.

81 Complete HOIP- or HOIL-1-deficiency causes embryonic lethality due to TNF-induced vascular

82 defects $(21,25)$. The loss-of-function SHARPIN mutant mice, $c p d m$, are viable but succumb to

83 severe dermatitis from approximately 6 weeks of age $(26,27)$, primarily due to sensitization to 
84 TNF-induced cell death via apoptosis or necroptosis $(10,21-23)$. Roles for LUBAC in

85 lymphocyte differentiation, activation and survival (e.g. $(20,28,29))$ have also been reported.

86 Key questions in the field remain how complete or partial loss of LUBAC function impacts

87 various tissues and how these defects influence inflammatory and immune pathology $(17,18)$.

89 Given the importance of TNFRSF signaling in TEC and thymic tolerance $(7,9,30,31)$, we 90 investigated whether LUBAC function was required for TEC development and homeostasis.

91 Conditional ablation of HOIP or HOIL-1 in TEC caused severe thymic atrophy and T cell

92 deficiency. HOIL-1 was required for the development of the thymic medulla in young mice and

93 the maintenance of cTEC in adults. Thymic atrophy and the demise of HOIL-1-deficient TECs

94 was driven in part by caspase-8/MLKL-driven apoptosis/necroptosis; blockade of this process

95 restored cortical and medullary microenvironments and thymic $\mathrm{T}$ cell production. Conversely,

96 only mild disruption of the thymic microenvironment was observed in SHARPIN-deficient mice,

97 confined to a defect in immature mTECs that was not related to cell death. These findings

98 identify LUBAC as an essential signaling hub with distinct roles in mTEC development, cTEC

99 survival and thymic function.

100

101 Results

102 LUBAC proteins HOIL-1 and HOIP in TECs are essential to maintain thymic function

103 We first assessed expression of the three LUBAC components in RNAseq data from TEC 104 subpopulations purified from young adult 8 week-old mice. TEC can be sub-divided into three 105 main populations: cTEC, MHC $\mathrm{II}^{\text {low }}$ mTEC (termed mTEC $^{\text {low }}$ ) that contain a mixture of 106 precursors and differentiated cells, and MHC II ${ }^{\text {high }}$ mTEC (termed mTEC $^{\text {high }}$ ) including cycling 
107 cells and the AIRE+ subset(30). All three known LUBAC components were transcribed in all 108 TEC subsets, with Rnf31 (encoding HOIP) relatively lower in mTEC ${ }^{\text {high}}$, while mTEC ${ }^{\text {low }}$ 109 expressed the highest levels of Sipll (encoding SHARPIN) (Figure 1A). To determine the roles 110 of LUBAC components in TECs in vivo, we generated mice with Foxn $1^{\text {Cre }}$-driven deletion of

111 Rbckl or Rnf31, hereafter termed Hoil-1 $1^{4 F o x n 1}$ and Hoip ${ }^{4 F o x n 1}$, respectively $(20,21,25)$ (specific

112 deletion confirmed in Figure S1A). Mice in both of these strains were viable, reproduced 113 normally and had no overt health problems. The role of SHARPIN in TEC development was 114 assessed in the spontaneous loss-of-function mutant $c p d m$ mouse strain $\left(S h^{c p d m / c p d m}\right)(26,27)$, 115 prior to the onset of inflammation. We observed a modest reduction in the thymic cellularity of $116 S h^{c p d m / c p d m}$ mice; however, TEC-specific loss of HOIL-1 or HOIP caused severe thymic atrophy 117 in adult mice (Figure 1B).

119 We tracked thymocyte differentiation Hoil-1 $1^{4 F o x n 1}$ mice by analyzing CD4 vs. CD8 expression 120 and observed loss in all major stages of T cell differentiation (Figure 1C, Figure S1B). Deeper 121 analysis of $\mathrm{CD}^{-} \mathrm{CD}^{-}$double negative (DN) precursor stages revealed a proportional block at the 122 DN3 stage of differentiation and numerical loss in all thymocyte precursor stages in Hoil-1 $1^{\text {LFoxn } 1}$ 123 mice (Figure S1C). Thymic Treg cell production in 8-week-old Hoil-1 $1^{\triangle F o x n 1}$ mice was virtually 124 extinguished in the severely atrophic thymi (Figure S1D). These data show that TEC-specific 125 HOIL-1 deletion led to severe thymic hypotrophy and markedly impaired T cell differentiation.

127 We then compared the thymic phenotype of young (Figure 1D) and young adult Hoip ${ }^{\Delta \text { Foxn } 1}$ mice 128 (Figure S1E-G) and found that they closely resembled that of Hoil-1 $1^{4 F o x n 1}$ mice, with severe 129 deficiency in all major thymocyte subsets. Thus, HOIP and HOIL-1 are critical in TECs for 
130 establishing or maintaining thymic function, consistent with the essential roles of these proteins

131 in LUBAC activity (21).

133 The thymic defects in adult Hoil $-1^{\triangle F o x n 1}$ mice caused T cell lymphopenia in peripheral lymphoid 134 tissues (Figure S2A). Both $\mathrm{CD}^{+}$and $\mathrm{CD}^{+} \mathrm{T}$ cells were diminished and, consistent with the 135 thymic atrophy, naïve $\mathrm{CD} 44^{\text {low }} \mathrm{CD} 62 \mathrm{~L}^{\text {high }}$ populations were particularly affected with 136 homeostatic expansion of $\mathrm{CD}_{122} 2^{+}$"virtual" memory cells (Figure 1E, F, Figure S2B, C). 137 Although the proportions of proliferating $\mathrm{Ki} 7^{+} \mathrm{T}$ cells and FOXP3 ${ }^{+}$Treg cells were increased in 138 8-week-old Hoil-1 $1^{\Delta F o x n 1}$ mice, the overall numbers were largely normal (Figure S2D, E). This 139 loss of naïve $\mathrm{T}$ cell populations yet maintenance of the virtual memory and regulatory subsets is 140 consistent with the greater reliance on thymic output of the former (32). These defects extended 141 to the TCR repertoire, with alterations in T cells expressing distinct TCR $\beta$ chains (Figure S2F).

143 We then assayed thymus size throughout ontogeny to determine whether HOIL-1 was required in

144 TECs for thymic development or homeostasis. Overall thymic cellularity immediately following 145 birth was normal in Hoil-1 $1^{A F o x n 1}$ mice but by day 3-4 mild thymic hypotrophy was evident 146 (Figure 1G). All major thymocyte subsets, including Treg cells, were diminished and DN1 and 147 DN3 precursor populations were reduced in 4-day-old Hoil-1 ${ }^{\text {SFoxn1 }}$ mice (Figure S3A-C). T cell 148 lymphopenia was not yet evident in the spleen, although a mild reduction in the proportion of 149 naïve CD4+ T cells was detected in 4-day-old Hoil-1 $1^{4 F o x n 1}$ mice (Figure S3D-F). Thymus size in 150 Hoil-1 $1^{\triangle F o x n 1}$ mice peaked at days 9-10 but then atrophied, with approximately 12-fold lower 151 thymic cellularity at 8 weeks of age compared to controls (Figure 1G). These data reveal a 152 differential requirement for LUBAC components in TEC maintenance, with HOIL-1 and HOIP 
153 essential for thymic function beyond the perinatal stage and the establishment of a normal naïve

154 T cell pool.

155

156 TECs require HOIL-1 or HOIP for their homeostasis

157 We next investigated the role of HOIL-1 or HOIP in TEC homeostasis at key time points. Low

158 numbers of TECs could be recovered from the atrophied thymi of aged-matched 13-week-old

159 Hoil-1 $1^{\Delta F o x n 1}$ (Figure 2A) and Hoip ${ }^{\Delta F o x n 1}$ mice (Figure 2B). We analysed the phenotype of those

160 TECs that could be recovered and found very similar profiles in both Hoil-1 ${ }^{\Delta F o x n 1}$ or Hoip ${ }^{\Delta F o x n 1}$

161 mice, with severe loss in the number of mTEC (Ly51 UEA-1 ${ }^{+}$) (Figure 2C-F). Examination of

162 the thymic architecture of 8 -week-old Hoil-1 $1^{\Delta F o x n 1}$ or Hoip ${ }^{4 F o x n 1}$ mice revealed extensive

163 disruption of cortical and medullary regions (labelled with anti-keratin-8 versus anti-keratin-

164 5/UEA-1), including the AIRE $^{+}$compartment, which had almost disappeared (Figure 2G-L).

165 The loss of TEC in adult Hoil-1 $1^{4 F o x n 1}$ and Hoip ${ }^{4 F o x n 1}$ mice was characterized by large epithelial

166 cell-free areas and prominent $\mathrm{ERTR}^{+}$fibroblastic remodeling (Figure 2G-L). These data

167 indicate that HOIL-1 and HOIP are required for the differentiation and/or homeostasis of all

168 major TEC subpopulations in the adult thymus. The identical impact of TEC-specific HOIL-1- or

169 HOIP-deficiency on the thymus and TEC phenotype is consistent with observations in other

170 tissues and the complete ablation of LUBAC-mediated linear ubiquitination caused by loss of

171 either protein $(10,15,21)$.

172

173 We next sought to distinguish whether LUBAC activity was required for TEC differentiation or

174 homeostasis. We assayed TEC composition during the development of Hoil-1 $1^{\Delta F o x n 1}$ mice and

175 observed a slight reduction in total TEC numbers as early as E15.5 that worsened in 4-day 
176 postnatal mice (Figure 3A). Flow cytometric analysis was used to quantify the major 177 subpopulations of EpCAM ${ }^{+}$TECs: cTEC and mTEC, and the mTEC subpopulations mTEC ${ }^{\text {high }}$ 178 (Ly51 $\left.{ }^{-} \mathrm{UEA}-1^{+} \mathrm{MHCII}^{+} \mathrm{CD} 80^{\text {high }}\right)$ and mTEC $^{\text {low }}\left(\mathrm{Ly} 51 \mathrm{UEA}-1^{+} \mathrm{MHCII}^{+} \mathrm{CD} 80^{\text {low } /}\right.$ ) cells that 179 become apparent in day 4 mice (Figure 3B-D). We found a severe deficit in mTEC in embryonic 180 and neonatal thymi from Hoil- $1^{4 \text { Foxn1 }}$ mice (Figure 3B-D), including the tolerogenic AIRE $^{+}$ 181 population (Figure 3E). The proportion of Ki67 $7^{+}$TECs was increased in E15.5 and day 4 Hoil$1821^{\Delta F o x n 1}$ mice, suggesting specific loss of non-proliferating TECs and/or compensatory 183 proliferation of remaining cells (Figure 3F, G). Therefore, although TECs from Hoil-1 $1^{\text {SFoxn } 1}$ 184 mice were capable of proliferation and differentiation into the major TEC subpopulations, they 185 were unable to maintain normal numbers. By contrast, relatively normal numbers of cTECs were 186 recovered at these stages (Figure 3C, D).

188 The thymic architecture of neonatal Hoil-1 $1^{4 F o x n 1}$ mice was also perturbed. Although the 189 distribution of ERTR $7^{+}$fibroblasts was comparable, medullary regions (labelled with anti190 keratin-5, UEA-1 and AIRE) were fewer and smaller in Hoil-1 $1^{\Delta F o x n 1}$ mice at day 4 (Figure 3H191 J). Consistent with the flow cytometric analysis, a normal network of $\mathrm{K} 8^{+}$cTECs was apparent 192 in thymi from neonatal Hoil-1 $1^{\Delta F o x n 1}$ mice (Figure 3H). We conclude that HOIL-1 is not required 193 for mTEC differentiation per se but is essential for the expansion and maintenance of all mTEC 194 subpopulations in the perinatal thymus. Furthermore, HOIL-1-mediated signals are not required 195 for the early differentiation and expansion of cTECs yet is required for their maintenance and 196 thymic function later in life (Figure 1, 2). 
199 To explore how the loss of LUBAC function leads to these outcomes, we performed RNAseq 200 analysis on FACS-purified cTEC and MTEC $^{\text {high }}$ from 2-week-old Hoil-1 ${ }^{l o x} /$ lox (control) and Hoil-

$2011^{4 F o x n 1}$ mice. We selected this age because: 1) it immediately precedes severe thymic atrophy, 202 therefore the relevant transcriptional changes should be underway, 2) sufficient numbers of TEC 203 could be recovered and 3) the relative expression profiles of the LUBAC components was 204 equivalent to young adult mice (Figure S4A). Visualization of the relationships among the 205 populations in a multi-dimensional scaling plot showed: (1) that the 3 biological replicates 206 clustered together closely, indicating low experimental variability, (2) the first dimension 207 distinguished cTEC from mTEC $^{\text {hi }}$, and (3) the second dimension distinguished the transcriptional 208 impact of Hoil-1-deficiency (Figure 4A). Large transcriptional changes were caused by HOIL 209 loss in cTEC and mTEC $^{\text {high }}$, with $\sim 3,000$ and $\sim 5,700$ genes reaching the thresholds for statistical 210 significance, although these generally had modest overall expression levels (log expression) or

211 fold-changes (Figure 4B). KEGG pathway analyses of differentially expressed genes revealed 212 enrichment in those associated with cell adhesion, ECM interaction and various signaling 213 pathways in cTECs, including several metabolic pathways and cell cycle regulators in mTEC $^{\text {hi }}$ 214 (Figure S4B, C). Interestingly, we observed enrichment of genes involved in regulation of cell 215 projection organization and morphology among HOIL-1 induced transcripts in cTEC (Figure S4 216 D-F); processes recently implicated in thymic regeneration from aged-related involution (33).

218 LUBAC-dependent cell signaling can be required to prevent aberrant cell death via caspase-8219 dependent apoptosis and/or by MLKL-dependent necroptosis, depending on the cell type (20-22, $22024,29,34)$. Hierarchical clustering of the transcriptional profiles of genes involved in receptor221 mediated programmed cell death was visualized using heatmaps (Figure 4C). These clearly 
222 distinguished TEC subsets from the two genotypes, indicating that substantial differences in this

223 pathway were induced by the loss of HOIL-1 (Figure 4C). Among these changes, the

224 upregulation of Mlkl and Casp 8 was a distinguishing feature of cTECs and mTEC ${ }^{\text {high }}$ cells

225 isolated from 2-week-old Hoil-1 $1^{4 F o x n I}$ mice. These findings suggest that the loss of HOIL-1 in

226 TECs had sensitized them to MLKL-dependent necroptosis and/or caspase-8-driven apoptosis

227 just prior to the onset of severe thymic atrophy.

228

229 To test whether the TEC defects observed in HOIL-1-deficient mice were caused by the 230 induction of cell death, we generated Hoil-1 ${ }^{4 F o x n I} \mathrm{Casp}^{-/-} M l k l^{--}$mice in which both apoptotic and

231 necroptotic pathways are non-functional (35). We first established that young Casp $^{-/ /} \mathrm{Mlkl}^{-/}$

232 mice had normal TN, DP and SP thymocyte differentiation and splenic $\mathrm{T}$ cell homeostasis

233 (Figure 4D, E, S5), extending on previous analyses $(20,35)$ and isolating any phenotypes

234 observed in the compound mutants to changes in the TEC compartment. In striking contrast to 235 the severe thymic atrophy and $\mathrm{T}$ cell lymphopenia observed in Hoil-1 ${ }^{4 F o x n 1}$ mice, Hoil$2361^{4 F o x n I} \operatorname{Casp~}^{-/-} \mathrm{Mlkl}^{-/-}$mice had normal thymic cellularity and near complete restoration of the 237 peripheral $\mathrm{T}$ cell population (Figure 4D, E). This finding indicates that the combined loss of 238 Caspase-8 and MLKL prevented the thymic atrophy observed in adult Hoil-1 ${ }^{4 F o x n I}$ mice.

240 Interestingly, the rescue of thymic function was driven by only partial restoration of the thymic 241 microenvironment. Hoil- $1^{\text {lox/lox }} \mathrm{Casp}^{8^{-/}} \mathrm{MlkT}^{-/}$control mice had a reduction in overall TEC 242 number compared to Hoil-1 $1^{\text {lox/lox }}$ control mice due to loss of mTEC (Figure 4F, G). TEC number 243 was further decreased in Hoil-1 ${ }^{4 F o x n l} \operatorname{Casp~}^{-/-} \mathrm{Mlkl}^{-1-}$ mice, yet was higher than the atrophied 244 thymus of Hoil-1 $1^{4 F o x n l}$ mice, suggesting only a portion of TEC were rescued (Figure 4F). This 
245 rescue was accounted for mainly by increased mTEC, although there was a trend (not

246 statistically significant) towards higher cTEC in Hoil-1 ${ }^{\Delta F o x n 1} \mathrm{Casp}^{-/ /} \mathrm{Mlkl}^{-/-}$compared to Hoil-

$2471^{\Delta F o x n 1}$ mice (Figure 4G, H). Immunofluorescent staining of thymic sections from 8-week-old

248 Hoil-1 $1^{\Delta F o x n 1} \operatorname{Casp~}^{-{ }^{--}} \mathrm{Mlkl}^{-{ }_{-}}$mice confirmed that the rescue of HOIL-1-deficient TEC was partial,

249 demonstrating small, isolated medullary islets composing a reduced area compared to the large,

250 confluent medulla of thymi from control mice (Figure 4I, J, S5F). In contrast, a normal,

251 confluent $\mathrm{K}^{+}$cTEC network and cortical microenvironment was observed (Figure 4I, J),

252 contrasting the near complete loss of these cells and regions in Hoil-1 $1^{\Delta F o x n 1}$ mice (Figure 2 E, F).

253 Therefore, caspase- 8 and MLKL deficiency restored the cortical microenvironment and thymic

254 lymphopoiesis in Hoil-1 $1^{\Delta F o x n 1}$ mice, but only partially restored the thymic medulla.

256 Collectively, these findings demonstrate that a broad transcriptional program is coordinated in

257 TEC by HOIL-1-mediated signals and that antagonism of TEC necroptosis/apoptosis within this 258 program is a critical mechanism supporting thymic function.

260 SHARPIN is required for normal mEC $^{\text {low }}$ compartment

261 The severe thymic atrophy observed in Hoil-1 $1^{\Delta F o x n 1}$ and Hoip ${ }^{\Delta F o x n 1}$ mice prompted us to also 262 explore the function of the third LUBAC component, SHARPIN, in TECs and thymic function.

263 To circumvent potentially confounding effects of the psoriasis-like inflammatory syndrome in 264 these $S h^{c p d m / c p d m}$ mice (26), we analyzed TECs and thymic function in $S h^{c p d m / c p d m}$ mice prior to 265 the development of dermatitis. Consistent with previous data (20), we recovered normal 266 proportions of DP thymocytes in $S h^{c p d m / c p d m}$ mice, indicating that no stress-related atrophy had 267 occurred. Nevertheless, mild thymic hypotrophy was accompanied by a trend towards lower 
268 TEC numbers (Figures 1B and 5A), with half the normal number of $\mathrm{mTEC}^{\text {low }}$ in $S h^{c p d m / c p d m}$ mice

269 (Figure 5B, C). The numbers of cTEC, mTEC ${ }^{\text {high }}$, AIRE ${ }^{+}$TECs and the proportions of

270 proliferating $\mathrm{Ki}^{+} 7^{+}$TECs were similar in controls and $S h^{c p d m / c p d m}$ mice (Figure 5C, S6A, S6B).

271 The observed mTEC ${ }^{\text {low }}$ defect was not recapitulated in $S h^{c p d m / c p d m} \rightarrow$ wt (Ly5.1) hematopoietic

272 chimeras (Figure S6C-F), indicating that the mTEC $^{\text {low }}$ defect was a primary consequence of the

273 loss of SHARPIN in the thymic stroma. Analysis of the thymic architecture of Sharpin ${ }^{c p d m / c p d m}$

274 mice revealed mild disruption of the thymic medulla compared to controls, although the location

275 and frequency of $\mathrm{AIRE}^{+}$TECs and ERTR7 ${ }^{+}$fibroblasts were comparable to controls (Figure 5D-

276 F). We conclude that SHARPIN-mediated signals are required specifically to maintain the

277 mTEC ${ }^{\text {low }}$ population.

278

279 SHARPIN is required to antagonize TNF-induced cell death in certain contexts (10). This pro280 survival activity is not dependent on NF- $\mathrm{BB}$ signaling but involves direct linear ubiquitination of

281 the TNFR1 signalling complex, recruitment of IKK complexes to phosphorylate RIPK1 and 282 prevent caspase-8-mediated apoptosis or RIPK3/MLKL-dependent necroptosis (10, 22-24). To

283 test whether the loss of $\mathrm{mTEC}^{\text {low }}$ in $S h^{c p d m / c p d m}$ mice was driven by TNF-induced, caspase-8-

284 dependent apoptosis or RIPK3- and MLKL-dependent necroptosis, we assayed for rescue of the

285 phenotype when these pathways were disabled. Genetic ablation of both caspase-8-driven

286 apoptosis and RIPK3/MLKL-dependent necroptosis in $S h^{\text {cpdm/cpdm }} \operatorname{Casp}^{+/-} \operatorname{Ripk}^{-/-}$and

$287 S h^{c p d m / c p d m} \operatorname{Casp}^{-/-} \mathrm{Mlkl}^{-/}$mice failed to rescue the loss of mTEC ${ }^{\text {low }}$ observed in $S h^{c p d m / c p d m}$ mice

288 (Figure 5G). Consistent with this finding, $S h^{c p d m / c p d m} T n f^{/-}$mice also exhibited the loss of 289 mTEC $^{\text {low }}$ (Figure 5H). These data indicate that cell death driven by TNF or other death ligands 290 was not the cause of the mTEC defect in $S h^{c p d m / c p d m}$ mice. Therefore, we conclude that LUBAC 
291 deprived of SHARPIN sustains sufficient activity to support TEC survival and thymic function

292 but cannot maintain a normal mTEC $^{\text {low }}$ population.

293 
295 The attachment of Met1-linked "linear" chains of ubiquitin to proteins has emerged as a key 296 regulator of $\mathrm{NF}-\mathrm{\kappa B}$ and cell death signaling in inflammation, cell survival and differentiation 297 (36). LUBAC is the only E3 ligase complex known to mediate this form of ubiquitination and it 298 is composed of SHARPIN, HOIL-1 and HOIP. The loss of HOIP or HOIL-1 completely 299 abolishes linear ubiquitination. SHARPIN deficiency only partially reduces this activity, with 300 residual HOIL-1/HOIP complexes sufficient to sustain some LUBAC function in NF- $\mathrm{BB}$-related 301 programs and the prevention of cell death induced by death ligands other than TNF $(10,12,14$, $30221,22,25,34)$. Given the critical roles of TNFR family members and NF- $\mathrm{kB}$ signaling in mTEC 303 differentiation and homeostasis (4), we tested the importance of LUBAC function in TECs. Our 304 data highlight essential roles for LUBAC signalling in mTEC development on one hand, and 305 cTEC survival in adulthood on the other.

307 Conditional ablation of either HOIL-1 or HOIP in TECs greatly diminished all mTEC subsets 308 and the formation of the medulla early in life. This phenotype resembles that observed in mice 309 with compound deficiency in the TNFRSF members RANK plus CD40 or LT $\beta$ R plus CD40 (7, 3109,37 ), or those with loss of the NF- $\mathrm{BB}$ signaling proteins NIK, TRAF6 or REL-B, where severe 311 loss of multiple mTEC subpopulations was observed (4). It is likely that the requirement for the

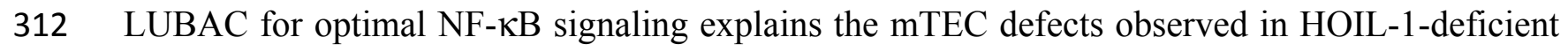
313 mice. However, we also found that Hoil-1 $1^{\Delta F o x n 1}$ mice succumbed to premature thymic atrophy 314 associated with loss of cTEC in adult animals, which appears to be a novel phenotype. These 315 cTEC defects are highly likely to cause the collapse of thymic function, since most thymocyte 316 proliferation is driven by this microenvironment. It is possible that LUBAC signals may be 
317 required for an aspect of cTEC function critical to the production of DP thymocytes, the loss of

318 which then feeds back to cause more severe defects in this compartment. Although Shen et al.

319 reported loss of cTEC in young $N i k^{A F o x n I}$ mice, this phenotype was likely a secondary

320 consequence of the severe autoimmune hepatitis and pneumonitis in these mice, resulting in the

321 stress-induced loss of DP thymocytes (38) required to support this thymic microenvironment. By

322 contrast, Hoil-1 $1^{4 F o x n 1}$ and Hoip ${ }^{4 F o x n 1}$ mice were overtly healthy and had no signs of stress-

323 induced DP thymocyte death.

324

325 The spontaneous upregulation of genes involved in apoptosis and necroptosis in HOIL-1-

326 deficient TEC hinted that the induction of aberrant cell death might drive their loss; a notion

327 supported by the rescue of the thymic cortex and thymic function in Hoil-1 ${ }^{4 \text { Foxn }}$ Casp $^{-/ /}$Mlkt $^{-/}$

328 mice. This finding is in accord with observations that LUBAC-deficiency in certain cell types

329 can predispose them to TNF-induced apoptosis (which is caspase-8-dependent) or necroptosis

330 (which is RIPK1/RIPK3/MLKL-dependent)(10, 21, 22, 25, 39). Although our genetic data

331 implicate aberrant cell death in the TEC loss, cortical collapse and thymic atrophy observed in

332 Hoil-1 $1^{4 F o x n 1}$ mice, only a modest increase in overall TEC number was observed in Hoil-

$3331^{4 F o x n l} \operatorname{Casp~}^{-/-} \mathrm{Mlkl}^{-{ }_{-}^{-}}$mice, despite restoration of a normal, confluent $\mathrm{K}^{+}{ }^{+}$cTEC network. This

334 observation may reflect a technical limitation of flow cytometric analysis of TEC, whereby the 335 recovery of cTEC greatly underestimates the total number of these cells, as established by 336 Sakata, et al. (40). Other approaches will be required to confirm whether loss of LUBAC 337 function primarily impacts cTEC survival in vivo. Alternatively (or in addition), defective 338 regulation of cTEC morphology may influence the atrophy observed in Hoil-1 ${ }^{4 F o x n I}$ mice. We 339 found changes in the expression of genes regulating cellular projections in cTEC from Hoil- 
$3401^{4 F o x n I}$ mice, reminiscent of features reported in thymic regeneration in aged mice that were 341 independent of cTEC numerical changes(33). While the precise mechanisms remain to be

342 determined, it is clear that the main lymphopoietic cTEC niches were restored in Hoil-

$3431^{4 F o x n l} \operatorname{Casp~}^{-{ }^{--}} \mathrm{Mlkl}^{-/-}$mice, uncovering a critical signaling axis in the cTEC essential for thymic

344 function.

345

346 We also found that the restoration of the medulla was not complete in $\mathrm{Hoil}_{-1}{ }^{4 \mathrm{Foxn} l} \mathrm{Casp}^{-/-} \mathrm{Mlkl}^{-{ }^{-}}$

347 mice, therefore it is likely LUBAC modulates other signals supporting TEC expansion and/or 348 survival. In this regard, we note our RNAseq analysis of TEC from Hoil-1 $1^{4 F o x n l}$ mice revealed

349 heightened transcription of $\operatorname{Trp} 53$ which, although required for TEC function (41), can also 350 activate cell death and senescence (42). Future studies will reveal how LUBAC activity 351 intersects with these pathways to impact TEC differentiation, survival and function.

353 Consistent with the subordinate role for SHARPIN in linear ubiquitination, thymi from $354 S h^{c p d m / c p d m}$ mice exhibited milder TEC defects. Although there was a reduction in $\mathrm{mTEC}^{\text {low }}$ in $355 S h^{c p d m / c p d m}$ mice compared to controls, all other major TEC subsets were normal. Thus, there 356 appears to be sufficient LUBAC activity in SHARPIN-deficient TEC to support near normal 357 thymic function and homeostasis. Since compound loss of TNF or Caspase- 8 plus RIPK3 or 358 Caspase-8 and MLKL did not restore the MTEC $^{\text {low }}$ compartment of $S h^{\text {cpdm/cpdm }}$ mice, we conclude 359 that the reduced LUBAC activity in $S h^{c p d m / c p d m}$ mice did not predispose these TECs to cell death.

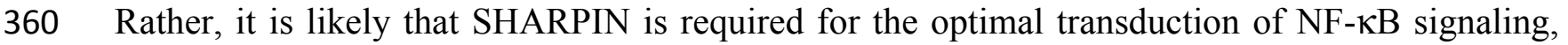
361 perhaps downstream of $\mathrm{CD} 40 / \mathrm{CD} 40 \mathrm{~L}$ interactions, which have previously been shown to 362 mediate mTEC $^{\text {low }}$ survival and/or expansion $(7,30)$. 
364 In conclusion, this study defines differential roles for LUBAC components in TECs that correlate 365 with their function in linear ubiquitination. These data reveal dual roles for LUBAC in the 366 development and maintenance of the thymic microenvironment. 
369 The Sharpin ${ }^{\text {cpdm/cpdm }}$ mutant mouse strain arose on a C57BL/6/Ka background (26) and these 370 mice were backcrossed twice onto a C57BL/6 background (22). The Foxn $1^{\text {cre }}$, Rnf $31^{\text {lox }}$, Rbck $^{\text {lox }}$,

$371 S h^{c p d m / c p d m} \mathrm{TNF}^{-/}, \mathrm{Sh}^{\mathrm{cpdm} / \mathrm{cpdm}} \mathrm{Casp}^{-/-} \mathrm{Mlkl}^{-/}$and $\mathrm{Sh}^{\mathrm{cpdm} / \mathrm{cpdm}} \mathrm{Casp}^{+/-} \mathrm{Ripk3}^{-/-}$were generated as

372 previously described $(20,22,25,43)$ and were maintained on a C57BL/6 background. No 373 randomisation or blinding of animals was performed for experiments. All mice were housed

374 under specific pathogen-free housing conditions according to the regulations of the Walter and 375 Eliza Hall Institute of Medical Research.

377 Thymus digestion

378 This procedure is described in detail elsewhere (44); briefly, the two thymic lobes were separated 379 and connective tissue was removed with forceps. Snips were made in each lobe with surgical 380 scissors and the fragments were agitated in $5 \mathrm{~mL}$ of RPMI-1640 medium with $25.96 \mathrm{mM}$ HEPES 381 with a wide-bore pipette tip. The supernatant was recovered and replaced by $1 \mathrm{~mL}$ of digestion 382 buffer (RPMI-HEPES supplemented with 0.5 Wunsch units Liberase TM (Roche) and DNase I 383 at $0.1 \%(\mathrm{w} / \mathrm{v})\left(\right.$ Sigma-Aldrich)). Thymic tissue was then digested at $37^{\circ} \mathrm{C}$ for 15 min with gentle 384 agitation after every 5 min. The supernatant was then replaced with $500 \mu \mathrm{L}$ of digestion buffer 385 and the digestion incubation was repeated for $15 \mathrm{~min}$. The single cell suspensions recovered as 386 Fractions 1 and 2 were stained with antibodies to analyze TEC phenotype and number.

\section{$388 \quad$ Flow cytometry}


389 Single-cell suspensions of lymphoid tissue were stained with various fluorochrome-conjugated 390 antibodies. Surface staining of TECs was performed using the following antibodies that were 391 made at The Walter and Eliza Hall Institute, unless otherwise stated. The TEC lineage depletion 392 cocktail consisted of antibodies against mouse CD16/32 (FcgR-block, clone 2.4G2), mouse 393 CD45 PerCP/Cy5.5 (clone 30-F11, Biolegend), mouse CD31 PerCP/Cy5.5 (clone 390, 394 Biolegend) and mouse TER119 PerCP/Cy5.5 (clone TER119, Biolegend). Other conjugates 395 included antibodies to mouse CD326 (EpCAM) APC/Cy7 (clone G8.8, Biolegend), H2-A/E 396 FITC or APC (clone M5/114.15.2), H2-A/E BV421 (clone M5/114.15.2, Biolegend), 397 biotinylated UEA-1 lectin (Vector labs, USA), mouse Ly51 PE or FITC (clone 6C3, Biolegend) 398 and CD80 BV421 (clone 16-10A1, Biolegend). Second step staining with streptavidin PE/Cy7 399 (BD Biosciences, USA) was used to detect biotinylated UEA1 (Vector Laboratories). Propidium 400 iodide (PI) or DAPI at a final concentration of $2.5 \mu \mathrm{g} / \mathrm{mL}$ was added to unfixed samples just 401 prior to data acquisition to label dead cells. Intracellular staining with antibodies against human 402 Ki67 FITC (clone MOPC-21, BD Pharmingen) and mouse AIRE FITC (clone 5H12) was 403 performed after fixation and permeabilization using the FoxP3 detection kit (eBioscience). 404 Lymphocytes were stained using antibodies of the following specificities: mouse TCR $\beta$ PE/Cy7 405 (H57.59.1, Biolegend), mouse CD4 APC (clone H129), mouse CD4 PerCP/Cy5.5 (GK1.5, 406 Biolegend), mouse CD8 APC/Cy7 or BV650 (clone 53-6.7, Biolegend), mouse CD25 PE or 407 BV510 (clone PC61, Biolegend), mouse CD44 PE or FITC (clone IM781), mouse CD122 PE 408 (clone TM- $\beta 1$ ), mouse CD62L APC/Cy7 (clone MEL-14, Biolegend) and mouse FOXP3 eFluor409450 (clone FJK-165, eBioscience). The immature thymocyte depletion cocktail contained 410 biotinylated antibodies against mouse NK1.1 (clone PK136, Biolegend), TER119 (TER119), 411 GR1 (clone RB6-8C5), Mac-1 (clone M1-170) and B220 (RA3-6B2), and they were detected 
412 with streptavidin BV786 (Biolegend). Screening of TCRV $\beta$ repertoire in the CD4 $4^{+}$and CD8 ${ }^{+}$

413 populations was performed with the mouse V $\beta$ TCR Screening Panel (BD Pharmingen). Samples

414 were acquired using Fortessa X20 (BD Biosciences) and LSR II analysers (BD Bioscience) and

415 data analyzed using FlowJo software 9.9 (TreeStar).

416 PCR

417 The floxed allele (in the absence of Cre) in sorted TECs $\left(\mathrm{CD} 45^{-} \mathrm{MHCII}^{+} \mathrm{EpCAM}^{+}\right)$, stromal cells $418\left(\mathrm{CD}^{-} \mathrm{EpCAM}^{-}\right)$and hematopoietic cells $\left(\mathrm{CD}^{+} 5^{+} \mathrm{EpCAM}^{-}\right)$from 3-4 week-old Hoil-1 ${ }^{\text {lox/lox }}$ and 419 Hoil-1 $1^{4 F o x n 1}$ mice was detected by using Hoil-1-Fwd 5'-ACC CTA GGC CTA GTC AGT GCA 420 AA-3' with Hoil-1-Rev-5'-AGG CTG TGG TCC ATT CTA GCC AT-3' producing 580bp band.

421 The conditions were: $96{ }^{\circ} \mathrm{C} 2 \mathrm{~min} ; 30$ cycles for $96{ }^{\circ} \mathrm{C} 20 \mathrm{~s}, 57{ }^{\circ} \mathrm{C} 20 \mathrm{~s}, 72{ }^{\circ} \mathrm{C} 1 \mathrm{~min} 20 \mathrm{~s}$ and 422 final extension of $72{ }^{\circ} \mathrm{C} 5 \mathrm{~min}$. The deleted allele (after Cre-mediated recombination) was 423 detected by using Fwd 5'- ATG GTC TAC AGA AGA AAA CAG GC-3' and Rev 5'-GGG 424 AGA TTC AGA CAA GGT TTC-3' producing 581bp. The conditions were: $94^{\circ} \mathrm{C} 4 \mathrm{~min} ; 30$ 425 cycles for $94^{\circ} \mathrm{C} 40 \mathrm{~s}, 55^{\circ} \mathrm{C} 30 \mathrm{~s}, 72^{\circ} \mathrm{C} 1 \mathrm{~min}$ and final extension of $72^{\circ} \mathrm{C} 5 \mathrm{~min}$.

426

\section{Immunohistology}

428 Thymi from adult (8 weeks) and neonatal (day 4) mice were isolated, embedded in Tissue-Tek 429 O.C.T compound (Sakura Finetek, U.S.A.) and snap frozen in a liquid nitrogen/isopentane

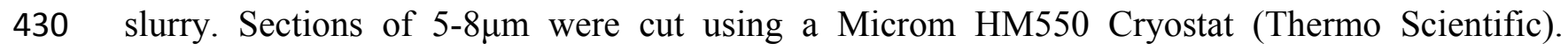
431 Sections were fixed in ice cold acetone (Merck) for $3 \mathrm{~min}$ and air-dried for $2 \mathrm{~min}$. Sections were 432 blocked with 5\% (v/v) goat serum in PBS with $0.1 \%$ Tween-20 (v/v) for 30 min at room 433 temperature before incubation with primary antibodies for $30 \mathrm{~min}$. Primary antibodies of the 434 following specificities were used: mouse K5 (Covance, clone Poly 19055), biotinylated mouse 
435 pan-keratin (LifeSpan BioSciences, clone Lu-5), biotinylated UEA-1 lectin (Vector labs, USA), 436 mouse AIRE-Alexa647 (clone 5H12), mouse K8 (clone Troma-I, DSHB) and ER-TR7 (provided

437 by Prof Richard Boyd, Monash University). Following three 5 min washes in PBS, sections were 438 incubated with appropriate secondary reagents (antibodies or streptavidin) conjugated to 439 fluorochromes (anti-rabbit IgG Alexa-555 (Life Technologies) and streptavidin FITC 440 (Invitrogen)) for $30 \mathrm{~min}$, counterstained with DAPI (Sigma-Aldrich), then mounted with 441 Vectashield (Vector labs). Images were collected using a LSM780 confocal microscope with Zen 4422012 SP2 (black) software v11.0 (Zeiss). Single optical sections and maximal intensity 443 projection images were processed for presentation using OMERO (45) or ImageJ (2.0.0). For 444 quantification of medullary area, 1 of 40 neighbouring sections, or every 1 of 20 for thymic from

445 adult Hoil-1 $1^{4 F o x n 1}$ mice, were selected as a representative section, stained with UEA-1(40) and 446 processed using ImageJ (2.0.0).

447

\section{$448 \quad$ RNA sequencing}

449 Thymi were pooled from 8- or 2-week-old WT mice and digested to isolate TECs (CD45$450 \mathrm{MHCII}^{+} \mathrm{EpCAM}^{+}$) (44). At the end of the digestion, fractions were pooled, enriched and purified 451 using anti-mouse CD45 microbeads (Miltenyi Biotec, Germany, Cat \# 130052301) and FACS 452 ARIA (BD). Cell pellets were snap frozen on dry ice and stored at $-80^{\circ} \mathrm{C}$. RNA was isolated 453 using the miRNeasy Micro Kit (Qiagen) with on column DNase digestion according to 454 manufacturer's instructions. First strand cDNA synthesis and cDNA amplification were 455 performed using the SMART-Seq ${ }^{\circledR}$ v4 Ultra ${ }^{\circledR}$ Low Input RNA Kit for Sequencing (Clontech 456 Laboratories) according to manufacturer's instructions. Complementary DNA (cDNA) libraries 457 were prepared and indexed separately using the Nextera ${ }^{\circledR}$ XT DNA Library Preparation Kit 
458 (Illumina) following manufacturer's instructions. Each indexed cDNA sample library was 459 quantified using the Agilent TAPE station and the Qubit ${ }^{\mathrm{TM}}$ DNA BR assay kit for Qubit 3.0® 460 Fluorometer (Life technologies). The indexed sample libraries were pooled and diluted to $1.5 \mathrm{pM}$ 461 for 75 base paired-end sequencing on a NextSeq 500 instrument using the v2 150 cycle High 462 Output kit (Illumina) according to the manufacturer's instructions.

463 Between 13 and 35 million read pairs were generated for each sample and reads were aligned to 464 the Mus musculus genome (mm10) using Rsubread (46). The number of read pairs overlapping 465 mouse Entrez genes was summarized using featureCounts and Rsubread's built-in NCBI gene 466 annotation. Low expressed genes were filtered out using edgeR's filterByExpr function (47).

467 Genes without current annotation were also filtered. Differential expression (DE) analyses were 468 undertaken using the edgeR and limma (48) software packages. Library sizes were normalized 469 using the trimmed mean of M-values (TMM) method (49). Sample quality weights were 470 estimated using voomWithQualityWeights (50)and differential expression was evaluated using 471 voom (51) with robust empirical Bayes estimation of the variances (52). Correlations between 472 repeated measurements from the same mouse were estimated using the duplicateCorrelation 473 method (53). The false discovery rate (FDR) was controlled below 0.05 using the method of 474 Benjamini and Hochberg. Over-representation of Gene Ontology (GO) terms and KEGG 475 pathways for the differentially expressed genes were identified using limma's goana and kegga 476 functions. Barcode plots illustrating the cross correlations between the cell types, and enrichment 477 of interested pathway genes were drawn using limma's barcodeplot function. Gene set 478 enrichment tests used the roast method (54). Heatmaps were drawn using limma's coolmap 479 function. Sequence data that support the findings of this study have been deposited with GEO 480 with the primary accession code GSE139898. 


\section{Hematopoietic reconstitution experiments}

483 Bone marrow reconstitution experiments were performed using recipient WT (C57BL/6-Ly5.1)

484 mice irradiated with 2x 550 RAD and reconstituted within 24 hours by intravenous injection with

$4855 \times 10^{6}$ of BM cells from donors of interest (all on a C57BL/6-Ly5.2 background). Reconstituted 486 mice were analyzed 6.5 weeks after reconstitution.

487

\section{Data analysis}

489 Statistical analyses were performed using Prism version 7. Experiments containing three or more 490 groups were analyzed using ANOVA followed by a Tukey's post-hoc test. Experiments with two 491 groups were analyzed with two-tailed Student's t-test. P-values $<0.05$ were considered as the 492 threshold for statistical significance for all statistical tests.

493

494 Author contributions

495 Conceptualization, R.J., A.S. and D.H.D.G.; Methodology, R.J., J.M.S., K.Z., A.S. and 496 D.H.D.G.; Investigation, R.J., J.M.S., M.H., W.A., K.Z., F.K. and D.H.D.G.; Resources, P.B., 497 C.H., J.R., H.W., J.S., A.S., D.H.D.G.; Writing - Original draft, R.J., J.M.S., and D.H.D.G.; 498 Writing - Review and editing, R.J., J.M.S., M.H., K.Z., A.S., H.W., J.S. and D.H.D.G. The 499 authors declare no conflict of interest. Correspondence and requests for materials should be 500 addressed to D.H.D.G. (dgray@wehi.edu.au).

501

502 Acknowledgments 
503 We gratefully acknowledge the Gray, Strasser and Herold laboratories and Dr Nieves Peltzer for

504 valuable feedback on this study. We thank the WEHI Flow Cytometry Laboratory and the Centre

505 for Dynamic Imaging; B Helbert, K Mackwell, C Young for mouse genotyping; G Siciliano, H

506 Marks, K Humphreys and S O'Connor for animal husbandry; and L Tai for technical assistance.

507 TROMA-I was deposited to the DSHB by Brulet, P./Kemler, R. (DSHB Hybridoma Product

508 TROMA-I). This work was supported by grants GNT0637353, GNT1049724, GNT1187367,

509 GNT1025594 and GNT1121325 and Fellowships 1090236 and 1158024 (for D.H.D.G.),

510 Principle Research Fellowship 1016701 (for J.S.), Senior Principal Research Fellowship

5111020363 (for A.S) from the Australian National Health and Medical Research Council and the

512 Leukaemia Research Foundation. R.J. was supported by MIRS and MIFRS from the University

513 of Melbourne. H.W. is supported by an Alexander von Humboldt Professorship Award, a

514 Wellcome Trust Investigator Award (214342/Z/18/Z), a Cancer Research UK programme grant 515 (A27323), a Medical Research Council UK Grant (MR/S00811X/1) and by the German 516 Research Foundation (DFG) in the context of SFB 1399 and SFB 1403. This work was made 517 possible through Victorian State Government Operational Infrastructure Support and Australian 518 Government NHMRC IRIISS. The authors have no conflicting financial interests.

\section{Conflicts of Interest Statement}

521 The authors declare no competing conflicts of interest. 


\section{References}

523 1. Petrie HT, Zuniga-Pflucker JC. Zoned out: functional mapping of stromal signaling

524 microenvironments in the thymus. Annu Rev Immunol. 2007;25:649-79.

525 2. Klein L, Kyewski B, Allen PM, Hogquist KA. Positive and negative selection of the T 526 cell repertoire: what thymocytes see (and don't see). Nat Rev Immunol. 2014;14(6):377-91.

527 3. Takaba H, Morishita Y, Tomofuji Y, Danks L, Nitta T, Komatsu N, et al. Fezf2 528 Orchestrates a Thymic Program of Self-Antigen Expression for Immune Tolerance. Cell. $529 \quad 2015 ; 163(4): 975-87$.

530 4. Abramson J, Anderson G. Thymic Epithelial Cells. Annu Rev Immunol. 2017;35:85-118.

531 5. Cosway EJ, Lucas B, James KD, Parnell SM, Carvalho-Gaspar M, White AJ, et al. 532 Redefining thymus medulla specialization for central tolerance. J Exp Med. 2017.

533 6. Sun SC. The non-canonical NF-kappaB pathway in immunity and inflammation. Nat Rev 534 Immunol. 2017.

535 7. Akiyama T, Shimo Y, Yanai H, Qin J, Ohshima D, Maruyama Y, et al. The tumor 536 necrosis factor family receptors RANK and CD40 cooperatively establish the thymic medullary 537 microenvironment and self-tolerance. Immunity. 2008;29(3):423-37.

538 8. Desanti GE, Cowan JE, Baik S, Parnell SM, White AJ, Penninger JM, et al. 539 Developmentally regulated availability of RANKL and CD40 ligand reveals distinct mechanisms 540 of fetal and adult cross-talk in the thymus medulla. J Immunol. 2012;189(12):5519-26.

541 9. Hikosaka Y, Nitta T, Ohigashi I, Yano K, Ishimaru N, Hayashi Y, et al. The cytokine 542 RANKL produced by positively selected thymocytes fosters medullary thymic epithelial cells 543 that express autoimmune regulator. Immunity. 2008;29(3):438-50. 
544 10. Gerlach B, Cordier SM, Schmukle AC, Emmerich CH, Rieser E, Haas TL, et al. Linear 545 ubiquitination prevents inflammation and regulates immune signalling. Nature. $546 \quad 2011 ; 471(7340): 591-6$.

547 11. Haas TL, Emmerich CH, Gerlach B, Schmukle AC, Cordier SM, Rieser E, et al. 548 Recruitment of the linear ubiquitin chain assembly complex stabilizes the TNF-R1 signaling 549 complex and is required for TNF-mediated gene induction. Mol Cell. 2009;36(5):831-44.

550 12. Tokunaga F, Nakagawa T, Nakahara M, Saeki Y, Taniguchi M, Sakata S, et al. 551 SHARPIN is a component of the NF-kappaB-activating linear ubiquitin chain assembly 552 complex. Nature. 2011;471(7340):633-6.

553 13. Draber P, Kupka S, Reichert M, Draberova H, Lafont E, de Miguel D, et al. LUBAC554 Recruited CYLD and A20 Regulate Gene Activation and Cell Death by Exerting Opposing 555 Effects on Linear Ubiquitin in Signaling Complexes. Cell Rep. 2015;13(10):2258-72.

556 14. Ikeda F, Deribe YL, Skanland SS, Stieglitz B, Grabbe C, Franz-Wachtel M, et al. 557 SHARPIN forms a linear ubiquitin ligase complex regulating NF-kappaB activity and apoptosis. 558 Nature. 2011;471(7340):637-41.

559 15. Kirisako T, Kamei K, Murata S, Kato M, Fukumoto H, Kanie M, et al. A ubiquitin ligase 560 complex assembles linear polyubiquitin chains. EMBO J. 2006;25(20):4877-87.

561 16. Yamanaka K, Ishikawa H, Megumi Y, Tokunaga F, Kanie M, Rouault TA, et al. 562 Identification of the ubiquitin-protein ligase that recognizes oxidized IRP2. Nat Cell Biol. $563 \quad 2003 ; 5(4): 336-40$.

564 17. Boisson B, Laplantine E, Dobbs K, Cobat A, Tarantino N, Hazen M, et al. Human HOIP 565 and LUBAC deficiency underlies autoinflammation, immunodeficiency, amylopectinosis, and 566 lymphangiectasia. J Exp Med. 2015;212(6):939-51. 
567 18. Boisson B, Laplantine E, Prando C, Giliani S, Israelsson E, Xu Z, et al. 568 Immunodeficiency, autoinflammation and amylopectinosis in humans with inherited HOIL-1 and 569 LUBAC deficiency. Nat Immunol. 2012;13(12):1178-86.

570 19. Liang Y, Seymour RE, Sundberg JP. Inhibition of NF-kappaB signaling retards 571 eosinophilic dermatitis in SHARPIN-deficient mice. J Invest Dermatol. 2011;131(1):141-9.

572 20. Teh CE, Lalaoui N, Jain R, Policheni AN, Heinlein M, Alvarez-Diaz S, et al. Linear 573 ubiquitin chain assembly complex coordinates late thymic T-cell differentiation and regulatory 574 T-cell homeostasis. Nat Commun. 2016;7:13353.

575 21. Peltzer N, Darding M, Montinaro A, Draber P, Draberova H, Kupka S, et al. LUBAC is 576 essential for embryogenesis by preventing cell death and enabling haematopoiesis. Nature. $577 \quad 2018 ; 557(7703): 112-7$.

578 22. Rickard JA, Anderton H, Etemadi N, Nachbur U, Darding M, Peltzer N, et al. TNFR1579 dependent cell death drives inflammation in Sharpin-deficient mice. Elife. 2014;3.

580 23. Kumari S, Redouane Y, Lopez-Mosqueda J, Shiraishi R, Romanowska M, Lutzmayer S, 581 et al. Sharpin prevents skin inflammation by inhibiting TNFR1-induced keratinocyte apoptosis. 582 Elife. 2014;3.

583 24. Lafont E, Draber P, Rieser E, Reichert M, Kupka S, de Miguel D, et al. TBK1 and 584 IKKepsilon prevent TNF-induced cell death by RIPK1 phosphorylation. Nat Cell Biol. 585 2018;20(12):1389-99.

586 25. Peltzer N, Rieser E, Taraborrelli L, Draber P, Darding M, Pernaute B, et al. HOIP 587 deficiency causes embryonic lethality by aberrant TNFR1-mediated endothelial cell death. Cell 588 Rep. 2014;9(1):153-65. 
589 26. HogenEsch H, Gijbels MJ, Offerman E, van Hooft J, van Bekkum DW, Zurcher C. A 590 spontaneous mutation characterized by chronic proliferative dermatitis in C57BL mice. Am J 591 Pathol. 1993;143(3):972-82.

592 27. Seymour RE, Hasham MG, Cox GA, Shultz LD, Hogenesch H, Roopenian DC, et al. 593 Spontaneous mutations in the mouse Sharpin gene result in multiorgan inflammation, immune 594 system dysregulation and dermatitis. Genes Immun. 2007;8(5):416-21.

595 28. Park Y, Jin HS, Lopez J, Lee J, Liao L, Elly C, et al. SHARPIN controls regulatory T 596 cells by negatively modulating the $\mathrm{T}$ cell antigen receptor complex. Nat Immunol. $597 \quad 2016 ; 17(3): 286-96$.

598 29. Webb LV, Barbarulo A, Huysentruyt J, Vanden Berghe T, Takahashi N, Ley S, et al. 599 Survival of Single Positive Thymocytes Depends upon Developmental Control of RIPK1 Kinase 600 Signaling by the IKK Complex Independent of NF-kappaB. Immunity. 2019;50(2):348-61 e4.

601 30. Gray DH, Seach N, Ueno T, Milton MK, Liston A, Lew AM, et al. Developmental 602 kinetics, turnover, and stimulatory capacity of thymic epithelial cells. Blood. 2006;108(12):377760385.

604 31. Williams JA, Zhang J, Jeon H, Nitta T, Ohigashi I, Klug D, et al. Thymic medullary 605 epithelium and thymocyte self-tolerance require cooperation between CD28-CD80/86 and 606 CD40-CD40L costimulatory pathways. J Immunol. 2014;192(2):630-40.

607 32. den Braber I, Mugwagwa T, Vrisekoop N, Westera L, Mogling R, de Boer AB, et al. 608 Maintenance of peripheral naive $\mathrm{T}$ cells is sustained by thymus output in mice but not humans. 609 Immunity. 2012;36(2):288-97. 
610 33. Venables T, Griffith AV, DeAraujo A, Petrie HT. Dynamic changes in epithelial cell 611 morphology control thymic organ size during atrophy and regeneration. Nat Commun. $612 \quad 2019 ; 10(1): 4402$.

613 34. Taraborrelli L, Peltzer N, Montinaro A, Kupka S, Rieser E, Hartwig T, et al. LUBAC 614 prevents lethal dermatitis by inhibiting cell death induced by TNF, TRAIL and CD95L. Nat 615 Commun. 2018;9(1):3910.

616 35. Alvarez-Diaz S, Dillon CP, Lalaoui N, Tanzer MC, Rodriguez DA, Lin A, et al. The 617 Pseudokinase MLKL and the Kinase RIPK3 Have Distinct Roles in Autoimmune Disease 618 Caused by Loss of Death-Receptor-Induced Apoptosis. Immunity. 2016;45(3):513-26.

619 36. Peltzer N, Walczak H. Cell Death and Inflammation - A Vital but Dangerous Liaison. 620 Trends Immunol. 2019;40(5):387-402.

621 37. Jenkinson SR, Williams JA, Jeon H, Zhang J, Nitta T, Ohigashi I, et al. TRAF3 enforces 622 the requirement for T cell cross-talk in thymic medullary epithelial development. Proc Natl Acad 623 Sci U S A. 2013;110(52):21107-12.

624 38. Shen H, Ji Y, Xiong Y, Kim H, Zhong X, Jin MG, et al. Medullary thymic epithelial NF625 kB-inducing kinase (NIK)/IKKalpha pathway shapes autoimmunity and liver and lung 626 homeostasis in mice. Proc Natl Acad Sci U S A. 2019;116(38):19090-7.

627 39. Shimizu Y, Taraborrelli L, Walczak H. Linear ubiquitination in immunity. Immunol Rev. $628 \quad 2015 ; 266(1): 190-207$.

629 40. Sakata M, Ohigashi I, Takahama Y. Cellularity of Thymic Epithelial Cells in the 630 Postnatal Mouse. J Immunol. 2018;200(4):1382-8. 
631 41. Rodrigues PM, Ribeiro AR, Perrod C, Landry JJM, Araujo L, Pereira-Castro I, et al.

632 Thymic epithelial cells require p53 to support their long-term function in thymopoiesis in mice.

633 Blood. 2017;130(4):478-88.

634 42. Aubrey BJ, Kelly GL, Janic A, Herold MJ, Strasser A. How does p53 induce apoptosis 635 and how does this relate to p53-mediated tumour suppression? Cell Death Differ. $6362018 ; 25(1): 104-13$.

637 43. Zuklys S, Gill J, Keller MP, Hauri-Hohl M, Zhanybekova S, Balciunaite G, et al. 638 Stabilized beta-catenin in thymic epithelial cells blocks thymus development and function. J 639 Immunol. 2009;182(5):2997-3007.

640 44. Jain R, Gray DH. Isolation of thymic epithelial cells and analysis by flow cytometry. 641 Curr Protoc Immunol. 2014;107:3 26 1-15.

642 45. Allan C, Burel JM, Moore J, Blackburn C, Linkert M, Loynton S, et al. OMERO: 643 flexible, model-driven data management for experimental biology. Nat Methods. 2012;9(3):24564453.

645 46. Liao Y, Smyth GK, Shi W. The R package Rsubread is easier, faster, cheaper and better 646 for alignment and quantification of RNA sequencing reads. Nucleic Acids Res. 2019;47(8):e47.

647 47. Chen Y, Lun AT, Smyth GK. From reads to genes to pathways: differential expression 648 analysis of RNA-Seq experiments using Rsubread and the edgeR quasi-likelihood pipeline. $649 \quad$ F1000Res. 2016;5:1438.

650 48. Ritchie ME, Phipson B, Wu D, Hu Y, Law CW, Shi W, et al. limma powers differential 651 expression analyses for RNA-sequencing and microarray studies. Nucleic Acids Res. $652 \quad 2015 ; 43(7): \mathrm{e} 47$. 
653 49. Robinson MD, Oshlack A. A scaling normalization method for differential expression 654 analysis of RNA-seq data. Genome Biol. 2010;11(3):R25.

655 50. Liu R, Holik AZ, Su S, Jansz N, Chen K, Leong HS, et al. Why weight? Modelling 656 sample and observational level variability improves power in RNA-seq analyses. Nucleic Acids 657 Res. 2015;43(15):e97.

658 51. Law CW, Chen Y, Shi W, Smyth GK. voom: Precision weights unlock linear model 659 analysis tools for RNA-seq read counts. Genome Biol. 2014;15(2):R29.

660 52. Phipson B, Lee S, Majewski IJ, Alexander WS, Smyth GK. Robust Hyperparameter 661 Estimation Protects against Hypervariable Genes and Improves Power to Detect Differential 662 Expression. Ann Appl Stat. 2016;10(2):946-63.

663 53. Smyth GK, Michaud J, Scott HS. Use of within-array replicate spots for assessing 664 differential expression in microarray experiments. Bioinformatics. 2005;21(9):2067-75.

665 54. Wu D, Lim E, Vaillant F, Asselin-Labat ML, Visvader JE, Smyth GK. ROAST: rotation 666 gene set tests for complex microarray experiments. Bioinformatics. 2010;26(17):2176-82. 
670 Figure 1: Early thymic atrophy and T cell defects in Hoil-1 ${ }^{4 F o x n 1}$ and Hoip ${ }^{\Delta \text { Foxn } 1}$ mice

671 (A) RNA-seq expression analysis of LUBAC components from cTECs, mTEC ${ }^{\text {hi }}$ and mTEC ${ }^{\text {low }}$

672 from 8-week-old WT mice. (B) Thymic cellularity of 8-week-old Hoil-1 ${ }^{4 F o x n 1}$ or Hoip ${ }^{\Delta \text { Foxn1 }}$, 6-

673 week-old $S h^{c p d m / c p d m}$, mice versus controls. (C) Flow cytometry plots of thymocyte CD4 vs. CD8

674 expression from 8-week-old Hoil-1 $1^{4 F o x n 1}$ mice and Hoil-1 ${ }^{\text {lox/lox }}$ controls, with cell numbers

675 quantified (left panel). (D) Thymocyte subset numbers in 3-week-old Hoip ${ }^{\text {lox/lox }}$ and Hoip ${ }^{\text {4Foxn1 }}$

676 mice. (E) Numbers of splenic $\mathrm{TCR} \beta^{+} \mathrm{CD} 4^{+}$and $\mathrm{TCR} \beta^{+} \mathrm{CD} 8^{+} \mathrm{T}$ cells from 8-week-old Hoil-

$6771^{\text {SFoxn } 1}$ and Hoil-1 $1^{\text {lox/lox }}$ mice. (F) Flow cytometry plots of CD44 vs. CD62L expression gated on

678 splenic $\mathrm{TCR}^{+} \mathrm{CD} 4^{+}$or $\mathrm{TCR}^{+} \mathrm{CD}^{+} \mathrm{T}$ cells from 8-week-old Hoil-1 $1^{\text {AFoxn1 }}$ mice and Hoil-1 ${ }^{\text {lox/lox }}$

679 controls. Graphs show the numbers of naïve $\left(\mathrm{CD} 44^{\text {low }} / \mathrm{CD} 62 \mathrm{~L}^{\text {high }}\right)$, effector $\left(\mathrm{CD} 44^{\text {high }} / \mathrm{CD} 62 \mathrm{~L}^{\text {low }}\right)$

680 and central memory (CD44 $\left.4^{\text {high }} / \mathrm{CD} 62 \mathrm{~L}^{\text {high }}\right) \mathrm{T}$ cells. (G) Thymic cellularity of control and Hoil-

$6811^{\Delta F o x n 1}$ mice at the indicated ages. The numbers in parentheses indicate the mean fold-change in

682 thymic cellularity (controls vs Hoil-1 $1^{4 \text { Foxn1 }}$ mice). All data are representative of at least two

683 independent experiments shown (except A) ( $\mathrm{n} \geq 3$ /group). Graphs show mean \pm SEM and groups

684 were compared with a Student's t test (two-sided, unpaired). ${ }^{*} \mathrm{p}<0.05,{ }^{*} \mathrm{p}<0.01 ; * * * \mathrm{p}<0.001$;

$685 * * * * \mathrm{p}<0.0001$ 
687 Figure 2: Loss of TECs and severe disruption of thymic architecture in Hoil-1 ${ }^{4 \text { Foxn } 1}$ and 688 Hoip $^{4 F o x n 1}$ mice.

689 TEC $\left(\mathrm{CD}^{-} 5^{-\mathrm{MHCII}^{+}} \mathrm{EpCAM}^{+}\right)$number from (A) 13-week-old Hoil-1 ${ }^{\text {lox/lox }}$ and Hoil-1 $1^{\text {SFoxnl }}$ mice 690 or (B) 13-week-old Hoip ${ }^{\text {lox/lox }}$ and Hoip ${ }^{\Delta F o x n 1}$ mice. Representative flow cytometry plots gated on 691 TECs from (C) 13-week-old Hoil-1 $1^{\text {lox/lox }}$ and Hoil-1 ${ }^{4 F o x n 1}$ mice or (D) 13-week-old Hoip ${ }^{\text {lox/lox }}$ and 692 Hoip $^{\triangle F o x n 1}$ mice showing Ly51 vs. UEA-1 expression. (E, F) Mean proportion and number of

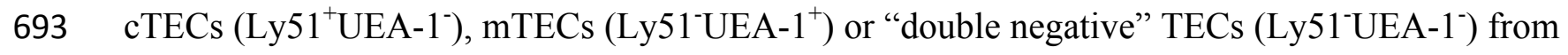
694 (E) 13-week-old Hoil-1 $1^{\text {lox/lox }}$ and Hoil-1 $1^{\Delta F o x n 1}$ mice or (F) 13-week-old Hoip ${ }^{\text {lox/lox }}$ and Hoip ${ }^{4 F o x n 1}$ 695 mice. (G-L) Immunofluorescence images of thymic sections from 8-9-week-old Hoil-1 $1^{\text {lox/lox }}$, 696 Hoil-1 $1^{4 F o x n 1}$, Hoip ${ }^{\text {lox/lox }}$ and Hoip ${ }^{4 F o x n 1}$ mice stained with anti-K8 and UEA-1 (G, J), anti-K5 and 697 anti-AIRE (H, K) and ER-TR7 and anti-PanK (I, L). Scale bars represent $200 \mu \mathrm{m}(\mathbf{G}, \mathbf{I}, \mathbf{J}, \mathbf{L})$ 698 and $20 \mu \mathrm{m}(\mathbf{H}, \mathbf{K}) . *$ and $* *$ represents epithelial-cell free regions. Data are representative of at 699 least two independent experiments ( $\mathrm{n} \geq 3$ /group). Graph bars indicate mean \pm SEM and groups 700 were compared with a Student's t test (two sided, unpaired). ${ }^{*} \mathrm{p}<0.05 ;{ }^{*} \mathrm{p}<0.01 ; * * * \mathrm{p}<0.001$; $701 \quad * * * * \mathrm{p}<0.0001$ 
703 Figure 3: HOIL-1 deficiency induces early loss of mTECs in Hoil-1 ${ }^{\text {AFoxn1 }}$ mice.

704 (A) TEC $\left(\mathrm{CD}^{-} 5^{-} \mathrm{MHCII}^{+} \mathrm{EpCAM}^{+}\right)$numbers from E15.5 or 4-day-old Hoil-1 ${ }^{\text {lox/lox }}$ and Hoil$705 \quad 1^{\Delta F o x n 1}$ mice. (B) Representative flow cytometry plots from thymic digests from individual E15.5 706 or 4-day-old Hoil-1 $1^{\text {lox/lox }}$ and Hoil-1 $1^{4 F o x n 1}$ mice showing Ly51 vs. UEA-1 gated on TECs (left and 707 middle panels) and CD80 vs. UEA-1 gated on mTECs (right panels). Graphs showing mean 708 proportions (top; of total TEC) and absolute numbers (bottom) of cTECs $\left(\mathrm{Ly} 51^{+} \mathrm{UEA}-1^{-}\right)$and 709 total mTECs $\left(\right.$ Ly51`UEA-1 ${ }^{+}$) from E15.5 (C) or cTECs, CD80 ${ }^{\text {hi }}$ mTECs and CD80 ${ }^{\text {lo/- }}$ mTECs 710 from 4-day-old (D) Hoil-1 $1^{\text {lox/lox }}$ and Hoil-1 $1^{4 F o x n 1}$ mice. (E) Representative flow cytometry plots of 711 MHC II vs AIRE expression gated on CD80 ${ }^{\text {hi }}$ mTECs from 4-day-old Hoil-1 $1^{l o x / l o x}$ and Hoil-

$7121^{\Delta F o x n 1}$ mice and mean cell numbers. (F) Representative histograms and (G) graphs showing 713 proportions of proliferating Ki67 ${ }^{+}$TECs. (H-J) Immunofluorescence images of thymic sections 714 from 4-day-old Hoil-1 $1^{l o x / l o x}$ and Hoil-1 $1^{4 F o x n 1}$ mice stained with anti-K8 and UEA-1 (H), anti-K5 715 and AIRE (I) and ER-TR7 and anti-PanK (J). Scale bars represent $200 \mu \mathrm{m}$ (H, J) and $20 \mu \mathrm{m}$ (I). 716 Graph bars indicate mean \pm SEM and experiments with two groups were compared with a 717 Student's t test (two sided, unpaired) 


\section{Figure 4: HOIL-1 is required to prevent TEC cell death}

720 (A) Multidimensional scaling (MDS) plot of RNAseq data from purified cTECs and mTEC ${ }^{\text {hi }}$

721 from 2-week-old Hoil-1 $1^{\text {lox/lox }}$ and Hoil-1 $1^{\Delta F o x n 1}$ mice, taking into account the top 500 most variable

722 genes between a given two samples. (B) Plots of the log-fold changes $\left(\right.$ Hoil-1 $1^{4 \text { Foxn } 1} /$ Hoil- $\left.1^{\text {lox/lox }}\right)$

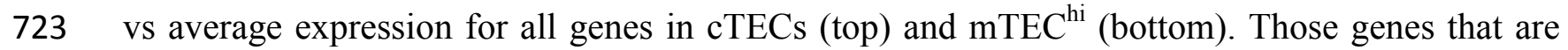

724 significantly upregulated (red) or downregulated (blue) in cell subsets from Hoil-1 $1^{\Delta \text { Foxn1 }}$ mice

725 when compared to Hoil-1 $1^{\text {lox/lox }}$ control mice are highlighted. (C) Heatmaps showing the

726 expression of genes involved in necroptosis (GO:0070266) in purified mTEC ${ }^{\text {hi }}$ and cTEC

727 subsets. (D) Graph of the mean thymic cellularity in 8-week-old Hoil-1 ${ }^{4 \text { Foxn } 1}$ Casp $^{-/-} \mathrm{Mlkl}^{-/-}$mice

728 vs controls. (E) Graph of the proportions of T cells among splenocytes in Hoil-1 $1^{\Delta F o x n 1} \operatorname{Casp} 8^{-/-}$

$729 M l k l^{-/-}$mice vs controls. (F-H) Graph of the total number of TECs $(\mathbf{F})$, mTECs $(\mathbf{G})$ or cTECs $(\mathbf{H})$

730 recovered from 8-week-old Hoil-1 ${ }^{\Delta F o x n 1} \operatorname{Casp}^{-/-} M l k l^{--}$mice vs controls. Data are combined from

731 three independent experiments ( $\mathrm{n} \geq 1-3$ /group). Graph bars indicate mean \pm SEM. (I, J)

732 Immunofluorescence images of thymic sections from 8-week-old Hoil-1 ${ }^{\text {lox/lox }}$ and Hoil-

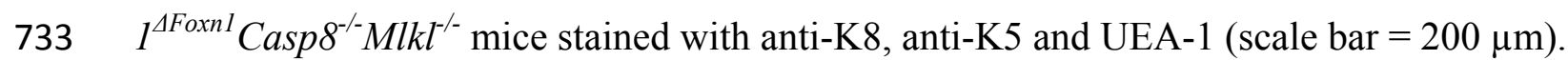


735 Figure 5: SHARPIN is required for mTEC $^{\text {lo }}$.

736 (A) $\mathrm{TEC}\left(\mathrm{CD}^{-} 5^{-} \mathrm{MHCII}{ }^{+} \mathrm{EpCAM}^{+}\right)$numbers from 6-week-old controls and $c p d m$ mice. (B)

737 Representative flow cytometry plots and (C) graphs showing proportions and absolute numbers

738 of mTEC $^{\text {hi }}\left(\mathrm{MHCII}^{\mathrm{hi}} \mathrm{Ly}^{5} 1^{\circ}\right)$ and $\mathrm{mTEC}^{\mathrm{lo}}$ mTECs $\left(\mathrm{MHCII}^{\mathrm{lo}} \mathrm{Ly} 51^{-}\right)$and cTECs $\left(\mathrm{MHCII}{ }^{+} \mathrm{Ly} 51^{+}\right)$.

739 Immunofluorescence images of thymic sections from 6-week-old $S h^{c p d m /+}$ and $S h^{c p d m / c p d m}$ mice

740 stained with (D) anti-K8 and UEA-1, (E) anti-K5 and AIRE and (F) ER-TR7 and anti-PanK.

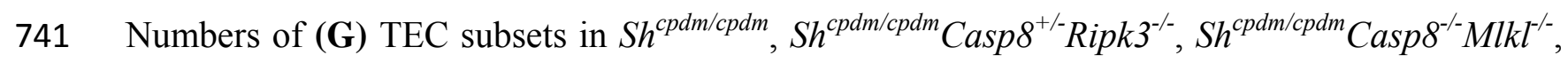

742 (H) $S h^{c p d m /+} T n f^{/}, S h^{c p d m / c p d m} T n f^{/}$. Graph bars indicate mean \pm SEM and groups were compared

743 with a Student's $t$ test (two sided, unpaired). NS, not significant $* \mathrm{p}<0.05 ;{ }^{* *} \mathrm{p}<0.01$; $* * *$

$744 \mathrm{p}<0.001 ; * * * * \mathrm{p}<0.0001$. The control group combines various combinations of genotypes $\left(\mathrm{Sh}^{+/+}\right.$,

$745 S h^{c p d m /+} ; \mathrm{n} \geq 3$ /group). Scale bars represent $100 \mu \mathrm{m}$ (D, F) and $20 \mu \mathrm{m}$ (E). 
Figure 1

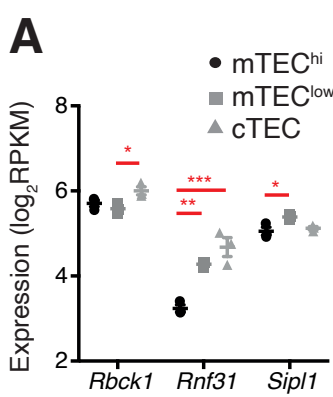

B

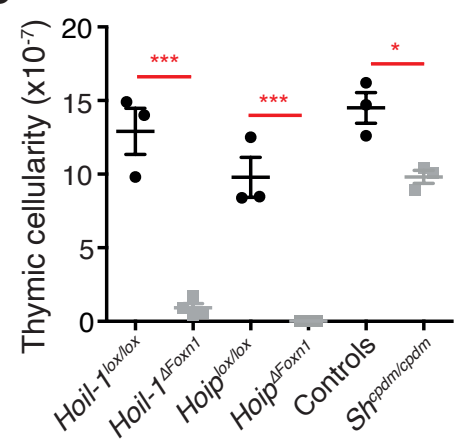

C
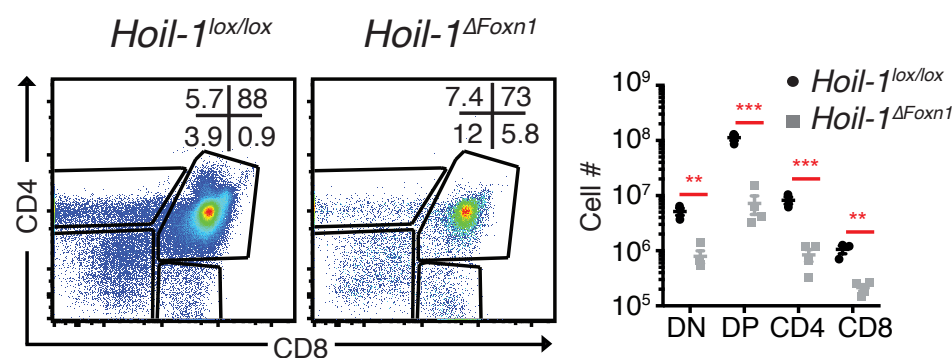

F
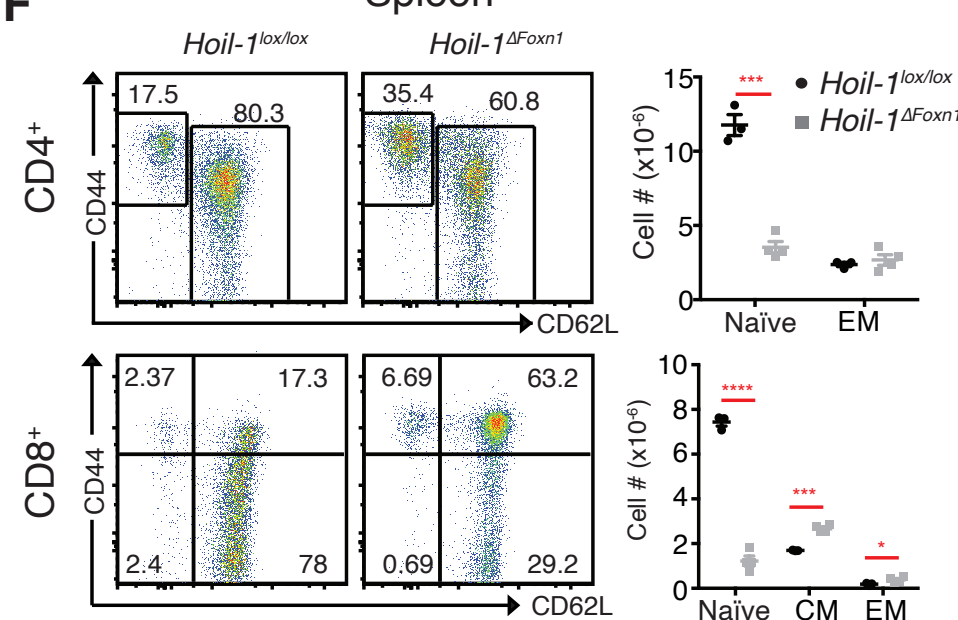

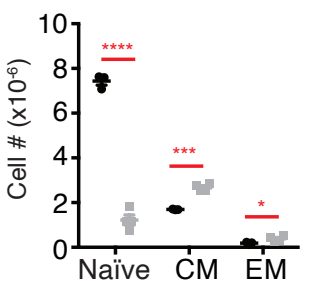

\section{E Spleen}

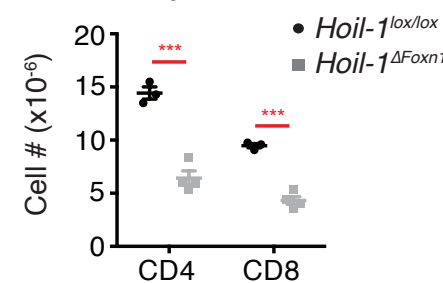

G

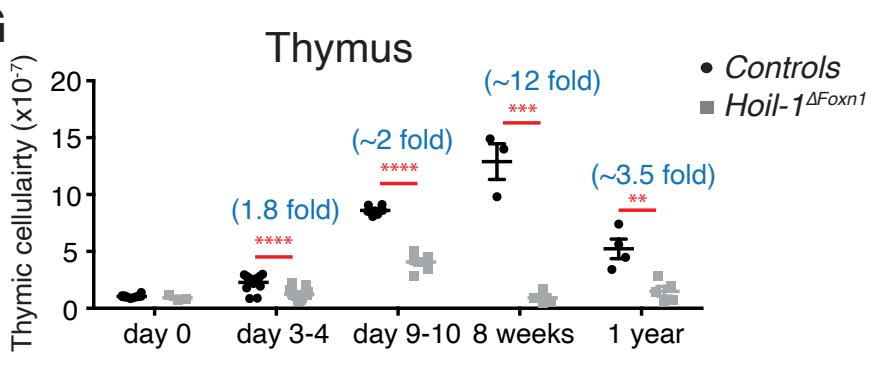



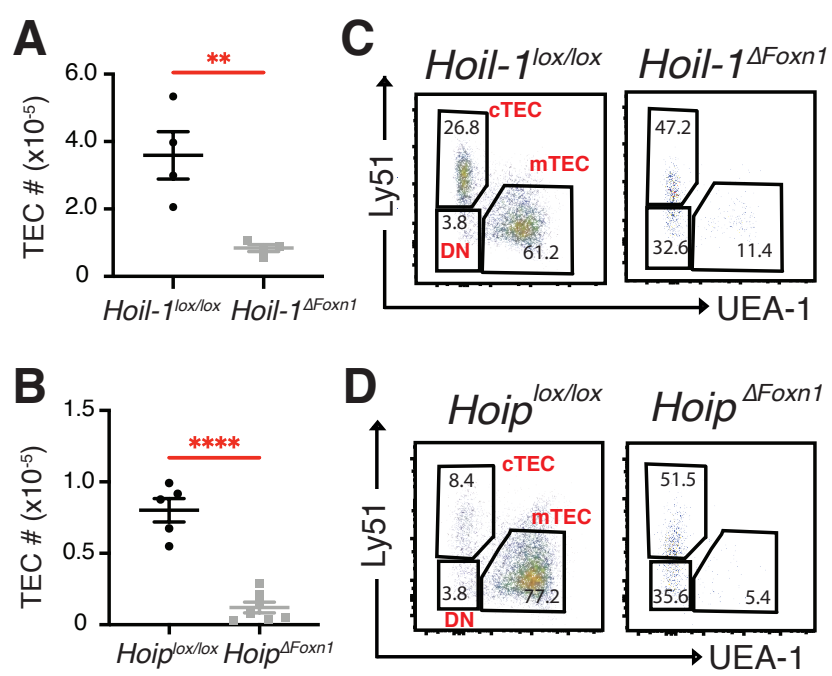

E

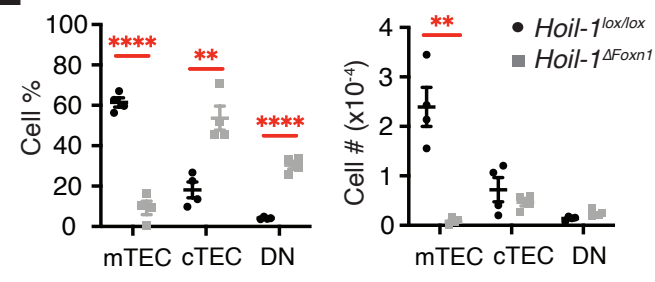

F

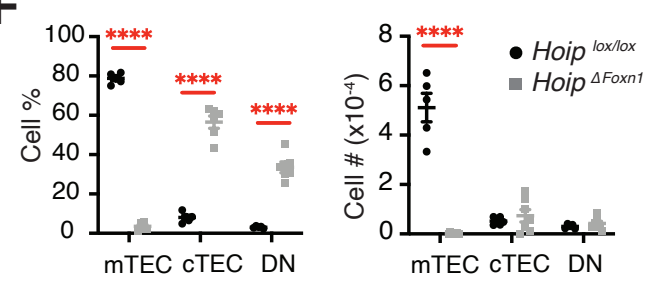

G K8 UEA-1

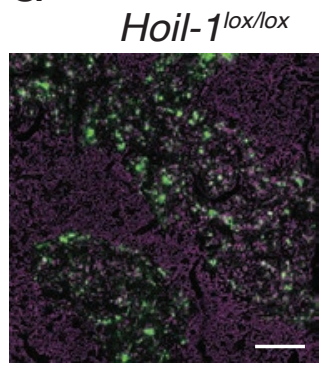

H

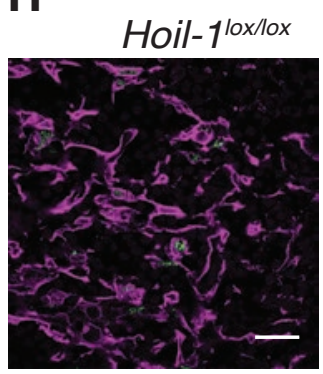

K5 AlRE

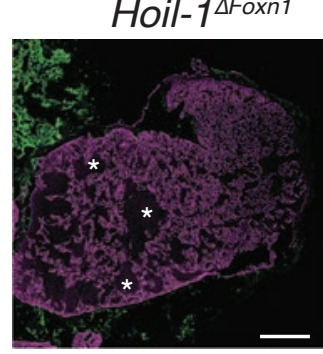

PanK ER-TR7

I

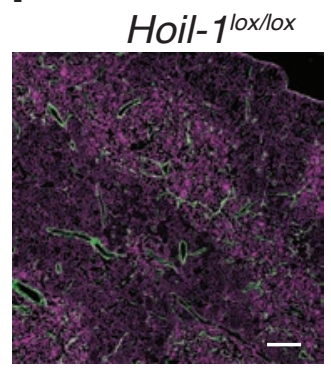

Hoil-1 ${ }^{\Delta F o x n 1}$

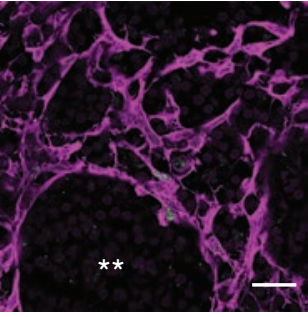

Hoil-1 ${ }^{\Delta F o x n 1}$

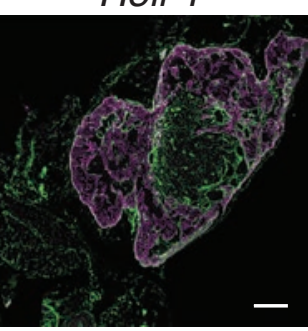

K8 UEA-1

Hoip-1 lox/lox Hoip-1 ${ }^{\triangle F o x n 1}$
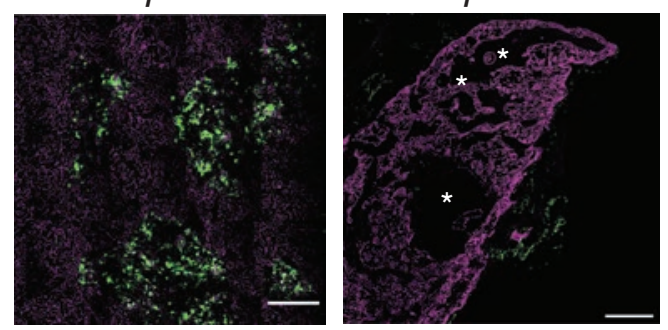

K K5 AIRE

Hoip-1 lox/lox Hoip-1 ${ }^{\Delta \text { Foxn1 }}$

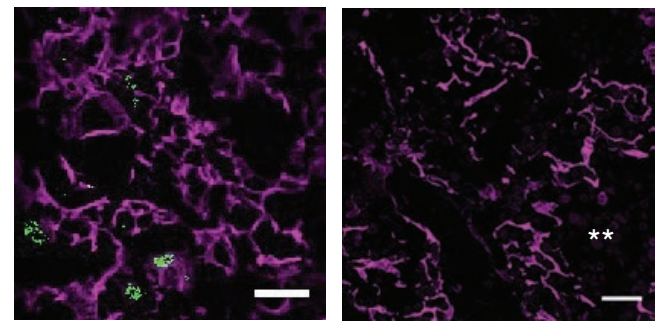

L PanK ER-TR7 Hoip-1 $10 x / / 0 x$ Hoip-1 ${ }^{\Delta F o x n 1}$

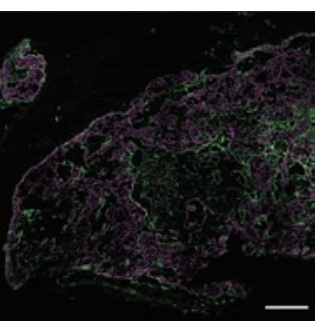


A

E15.5
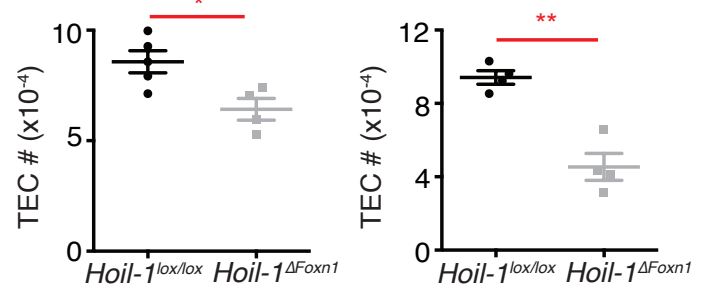

B
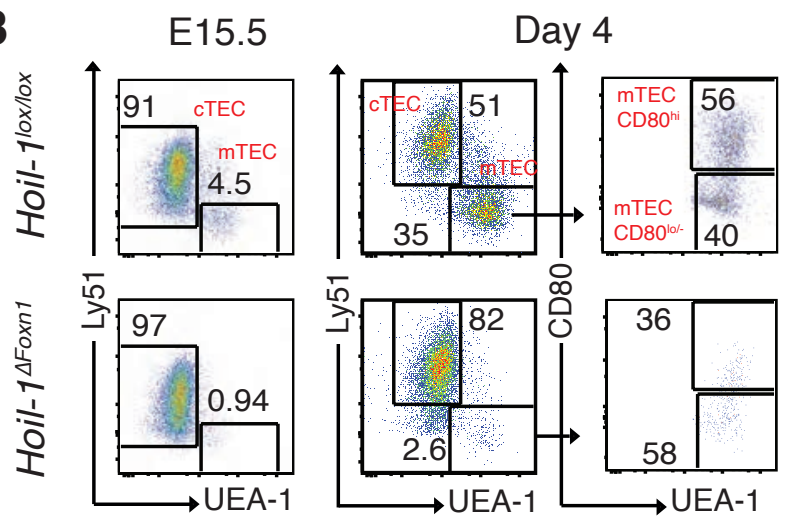

C

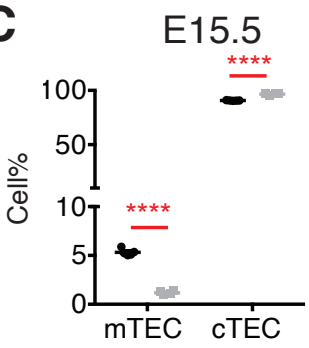

E15.5
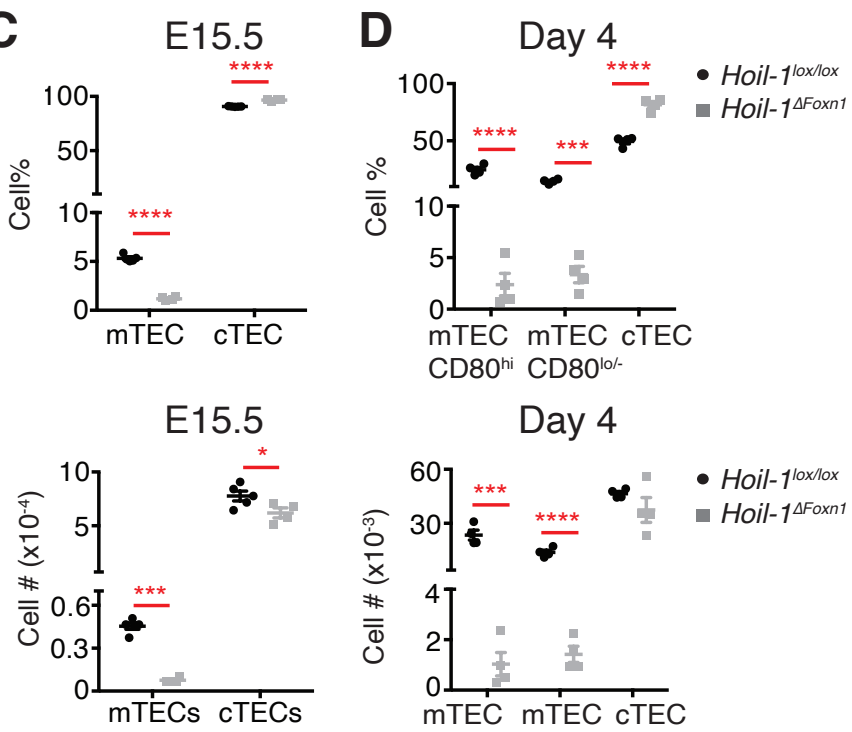

Day 4

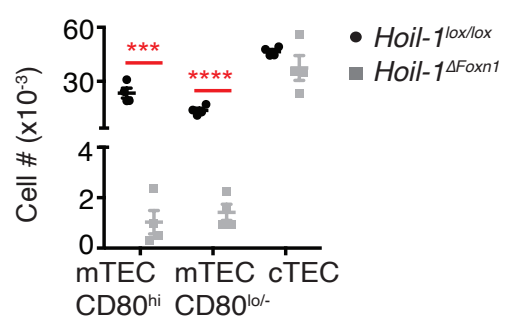

E

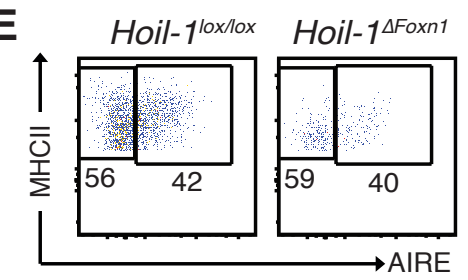

F

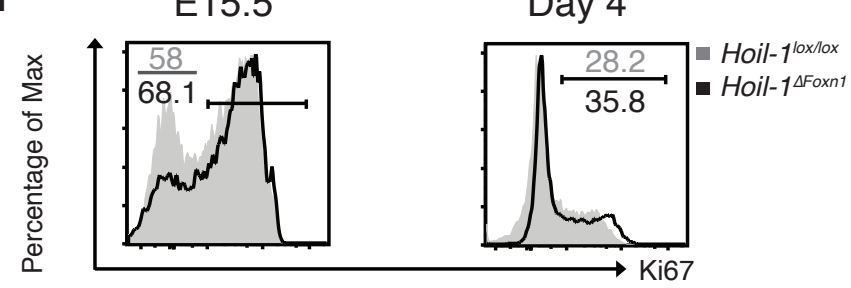

G

E15.5
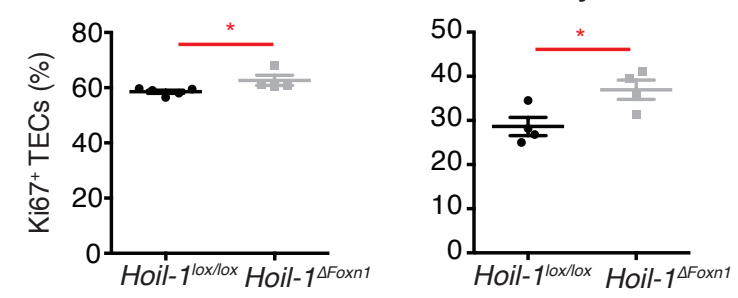

H

K8 UEA-1

Hoil-1lox/lox

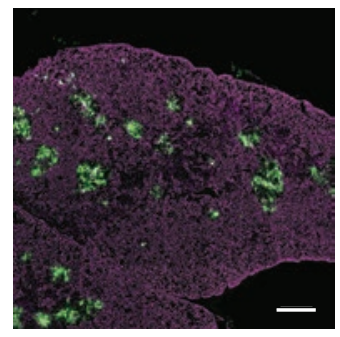

Hoil-1 ${ }^{\text {LFoxn } 1}$

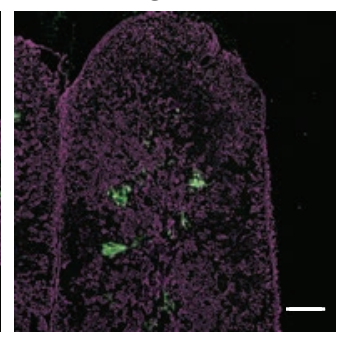

K5 AIRE

Hoil-1 10x/lox

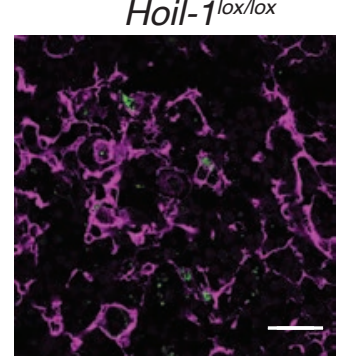

Hoil-1 ${ }^{\triangle F o x n 1}$

PanK ER-TR7

J

Hoil-1/ox/lox

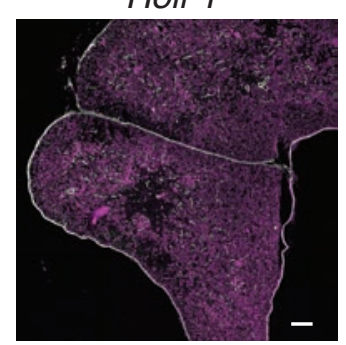

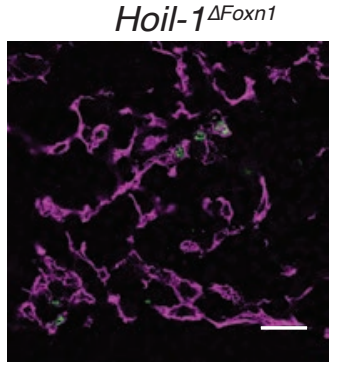

Hoil-1 ${ }^{\text {LFoxn1 }}$

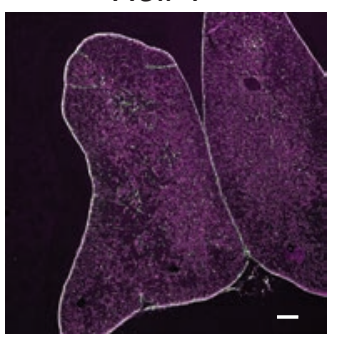




\section{Figure 4}
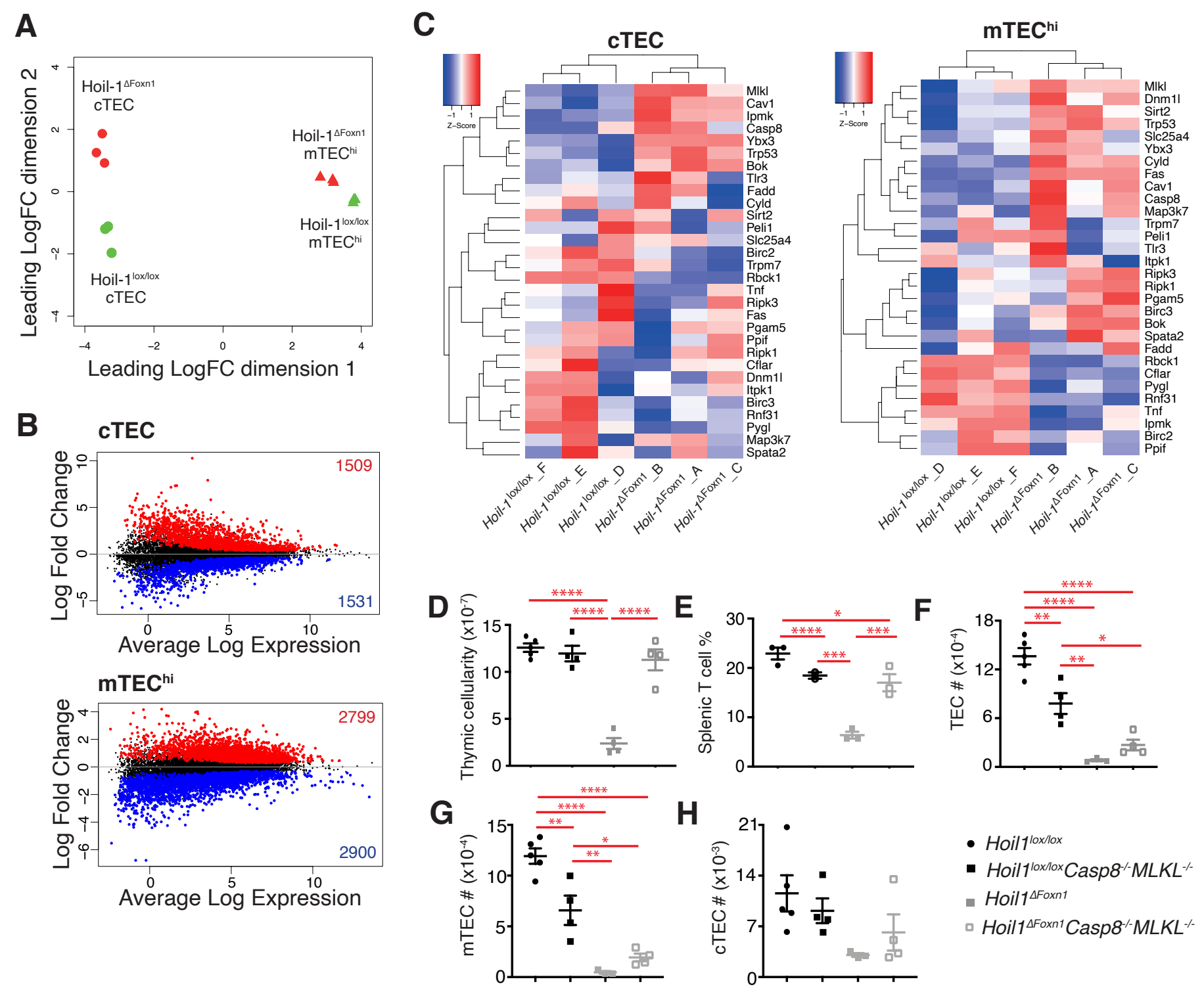

Hoil1 10x/lox

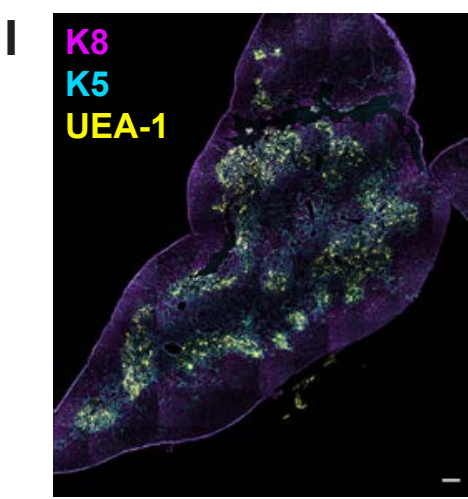

J

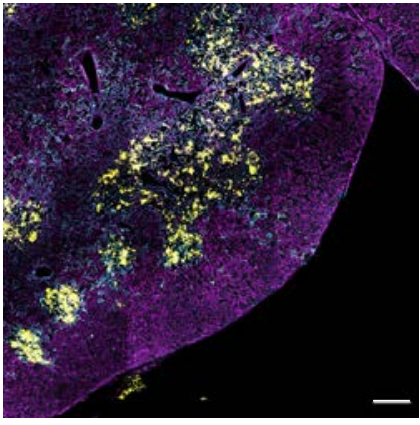

Hoil110x/lox $\mathrm{Casp}^{-/-} \mathrm{MLKL} \mathrm{KL}^{-/}$
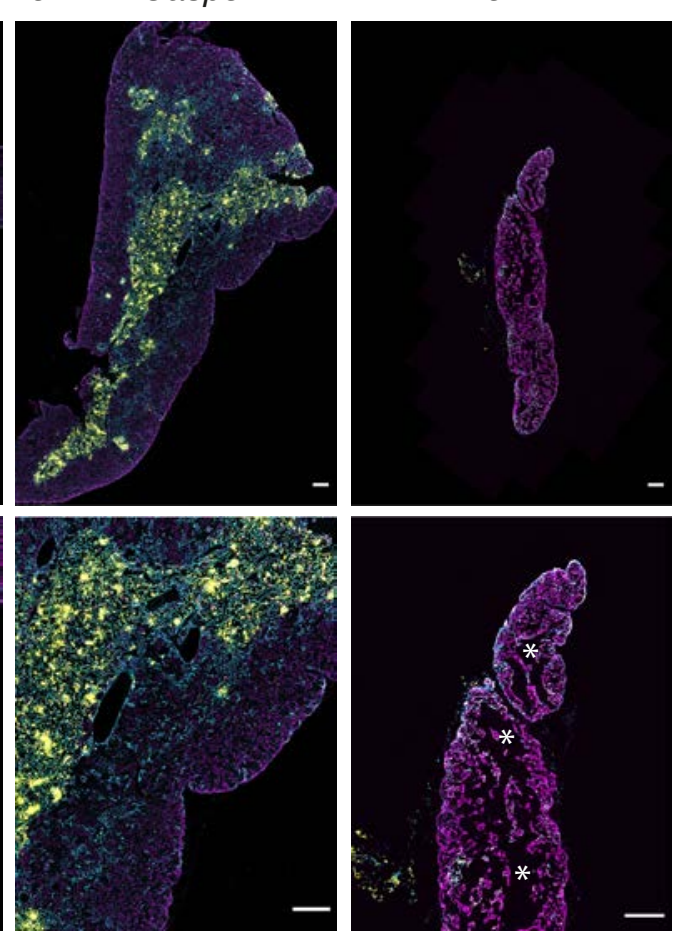
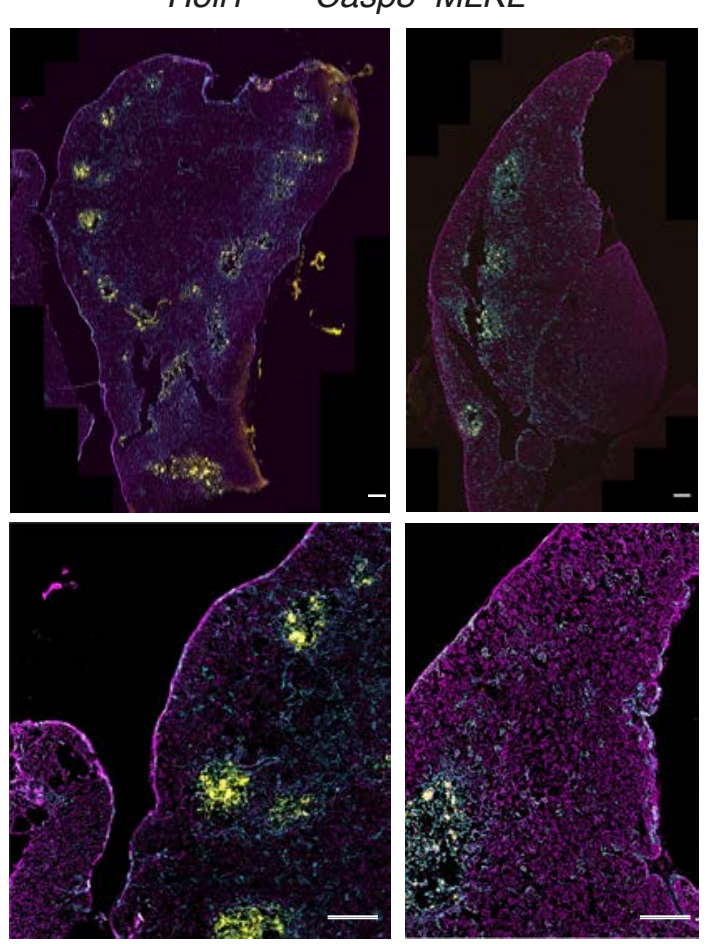


\section{Figure 5}
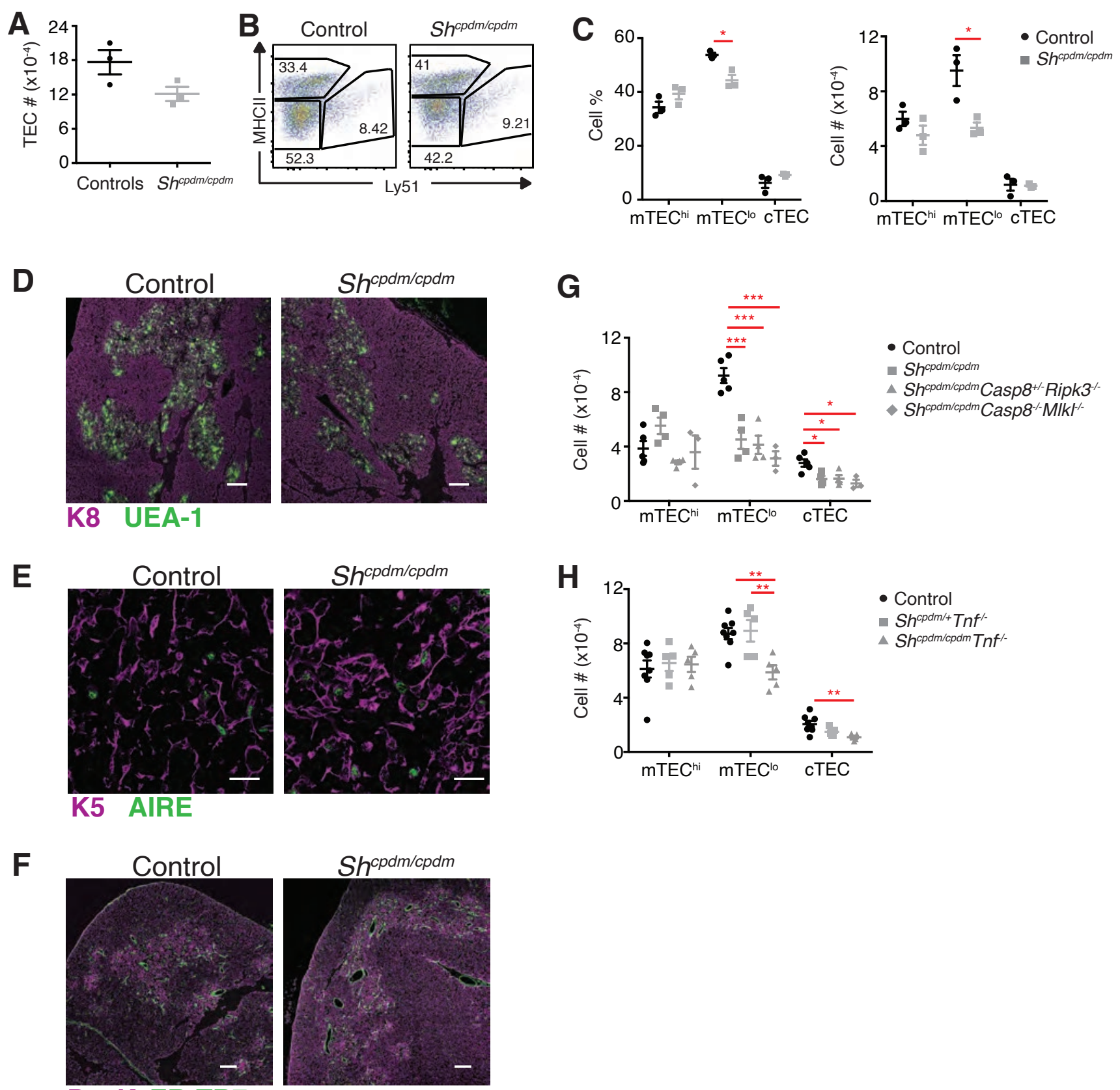

PanK ER-TR7 